JOURNAL OF THE

AMERICAN MATHEMATICAL SOCIETY

Volume 15, Number 1, Pages 167-184

S 0894-0347(01)00381-2

Article electronically published on September 24, 2001

\title{
STRONGLY TYPICAL REPRESENTATIONS OF THE BASIC CLASSICAL LIE SUPERALGEBRAS
}

\author{
MARIA GORELIK
}

\section{INTRODUCTION}

1.1. In PS1, I. Penkov and V. Serganova show that the category of representations of a basic classical Lie superalgebra $\mathfrak{g}$ of type I with a fixed typical central character is equivalent to the category of representations of the even part $\mathfrak{g}_{0}$ with a suitable central character. A similar result for type II was proven by I. Penkov in $[\mathrm{P}$ for "generic" central characters. The aim of this paper is to understand for which central characters such an equivalence holds. We also study a simplest example when such an equivalence fails to exist, but the corresponding category of representations of a Lie superalgebra still has a good description.

1.2. The basic classical Lie superalgebras were described by V. Kac in K1. These Lie superalgebras are the closest to the ordinary simple Lie algebras: for instance, they can be described by their Cartan matrices. The even part of a basic classical Lie superalgebra $\mathfrak{g}$ is a reductive Lie algebra. The centre $\mathcal{Z}(\mathfrak{g})$ is described in [K2], [S1], BZV]; it is isomorphic to a subalgebra of $\mathcal{S}(\mathfrak{h})^{W}$ and their fields of fractions coincide (here $\mathfrak{h}$ is a Cartan subalgebra of $\mathfrak{g}_{0}$ and $W$ is the Weyl group of $\left.\mathfrak{g}_{0}\right)$.

1.3. Let $\mathfrak{g}=\mathfrak{g}_{0} \oplus \mathfrak{g}_{1}$ be a basic classical Lie superalgebra, let $\tilde{\mathcal{U}}$ be its universal enveloping superalgebra, and let $\mathcal{Z}(\mathfrak{g})$ be the centre of $\widetilde{\mathcal{U}}$. Let $T$ be a special ghost element constructed in G1 - see 2.3. We call a maximal ideal $\tilde{\chi}$ of $\mathcal{Z}(\mathfrak{g})$ strongly typical if it does not contain $T^{2}$.

For a maximal ideal $\widetilde{\chi}$ of $\mathcal{Z}(\mathfrak{g})$ and a $\mathfrak{g}$-module $\widetilde{N}$ set $\widetilde{N}_{\tilde{\chi}}:=\left\{v \in \widetilde{N} \mid \widetilde{\chi}^{r} v=\right.$ $0, \forall r>>0\}$. For a fixed $\widetilde{\chi} \in \operatorname{Max} \mathcal{Z}(\mathfrak{g})$, denote by gr $\widetilde{\mathcal{C}}_{r}$ the category of graded $\mathfrak{g}$-modules $\widetilde{N}$ satisfying $\widetilde{\chi}^{r} \widetilde{N}=0$ and by gr $\widetilde{\mathcal{C}}_{\infty}$ the category of graded $\mathfrak{g}$-modules $\widetilde{N}$ satisfying $\widetilde{N}_{\tilde{\chi}}=\widetilde{N}$.

Consider $\mathfrak{g}_{0}$ as a purely even Lie superalgebra. Denote by $\mathcal{U}$ its enveloping algebra and by $\mathcal{Z}\left(\mathfrak{g}_{0}\right)$ the centre of $\mathcal{U}$. For a maximal ideal $\chi$ of $\mathcal{Z}\left(\mathfrak{g}_{0}\right)$ and a $\mathfrak{g}_{0^{-}}$ module $N$ define $N_{\chi}$ as above. For a fixed $\chi \in \mathcal{Z}\left(\mathfrak{g}_{0}\right)$, denote by $\operatorname{gr} \mathcal{C}_{r}$ the category

Received by the editors December 6, 2000.

2000 Mathematics Subject Classification. Primary 17B10, 17 B20.

Key words and phrases. Basic classical Lie superalgebra, Verma module.

The author was partially supported by TMR Grant No. FMRX-CT97-0100. Research at MSRI was supported in part by NSF grant DMS-9701755. 
of graded $\mathfrak{g}_{0}$-modules $N$ satisfying $\chi^{r} N=0$ and by $\operatorname{gr} \mathcal{C}_{\infty}$ the category of graded $\mathfrak{g}_{0}$-modules $N$ satisfying $N_{\chi}=\widetilde{N}$. In Section 3 we prove the following

1.3.1. Theorem. For a strongly typical $\tilde{\chi} \in \operatorname{Max} \mathcal{Z}(\mathfrak{g})$ the category $\operatorname{gr} \widetilde{\mathcal{C}}_{\infty}$ is equivalent to the category $\operatorname{gr} \mathcal{C}_{\infty}$ where $\chi$ is a suitable maximal ideal of $\mathcal{Z}\left(\mathfrak{g}_{0}\right)$. The equivalence is given by the functors

$$
\widetilde{N} \mapsto \widetilde{N}_{\chi}, \quad N \mapsto\left(\operatorname{Ind}_{\mathfrak{g}_{0}}^{\mathfrak{g}} N\right)_{\tilde{\chi}} .
$$

The restriction of these functors also provides the equivalence of $\operatorname{gr} \widetilde{\mathcal{C}}_{r}$ with $\operatorname{gr} \mathcal{C}_{r}$.

A maximal ideal $\chi \in \operatorname{Max} \mathcal{Z}\left(\mathfrak{g}_{0}\right)$ is suitable in the sense of the above theorem iff for some projective Verma $\mathfrak{g}$-module $\widetilde{M}$ satisfying $\widetilde{\chi} \widetilde{M}=0$, the $\mathfrak{g}_{0}$-module $\widetilde{M}_{\chi}$ is Verma and, moreover, one has $\widetilde{M}=\widetilde{\mathcal{U}} \widetilde{M}_{\chi}$. Note that if $\chi$ is a perfect mate for $\tilde{\chi}$ in the sense of [G2], then it fulfills these conditions. In [G2], Section 8, we prove the existence of a perfect mate $\chi$ for each strongly typical $\tilde{\chi} \in \operatorname{Max} \mathcal{Z}(\mathfrak{g})$. This allows one to describe the category gr $\widetilde{\mathcal{C}}_{\infty}$ corresponding to any strongly typical $\tilde{\chi}$ in terms of Theorem 1.3.1

The proof is based on the theorem stating that for a strongly typical $\tilde{\chi}$ the annihilator of a Verma $\mathfrak{g}$-module $\widetilde{M}$ satisfying $\widetilde{\chi} \widetilde{M}=0$ is equal to $\widetilde{\mathcal{U}}-$ see [G2].

An analogue of Theorem 1.3.1 for the basic classical Lie superalgebras of type I is proven in [PS1]; for "generic" $\tilde{\chi}$ in the type II case, it is proven in [P]. Both proofs are based on a realization of the categories $\operatorname{gr} \widetilde{\mathcal{C}}_{1}, \operatorname{gr} \mathcal{C}_{1}$ as categories of $D$-modules on the corresponding flag varieties.

1.4. Theorem 1.3.1 suggests a study of categories of representations of $\mathfrak{g}$ corresponding to a non-strongly typical character $\widetilde{\chi}$. Call such $\tilde{\chi}$ weakly atypical. If $\tilde{\chi}$ is not typical, the category $\widetilde{\mathcal{C}}_{\infty}$ has a very complicated structure - for instance, it contains non-trivial extensions of non-isomorphic finite-dimensional modules. An "intermediate" case, when $\widetilde{\chi}$ is typical but not strongly typical (it is possible only for the types $B(m, n), G(3))$, seems to be less complicated. We consider the case when $\mathfrak{g}=\mathfrak{o s p}(1,2 l)(B(0,2 l))$ and the maximal ideal is a "generic" weakly atypical (see the condition (101)). This means that the highest weight $\lambda$ of a highest weight module annihilated by $\mathfrak{m}$ belongs to exactly one of the hyperplanes $S_{\beta}, \beta \in \Delta_{1}^{+}$, where $S_{\beta}:=\left\{\mu \in \mathfrak{h}^{*} \mid(\mu+\rho, \beta)=0\right\}$.

It is convenient to substitute the maximal ideal $\mathfrak{m}$ of $\mathcal{Z}(\mathfrak{g})$ by the maximal ideal $\tilde{\chi}$ of the "ghost centre" $\widetilde{\mathcal{Z}}(\mathfrak{g})$ (see [G1] for a definition) which contains $\mathfrak{m}$. For $\mathfrak{g}=\mathfrak{o} \mathfrak{s p}(1,2 l)$ one has $\widetilde{\mathcal{Z}}(\mathfrak{g})=\mathcal{Z}(\mathfrak{g}) \oplus T \mathcal{Z}(\mathfrak{g})$ and the ideal $\widetilde{\chi}:=\mathfrak{m} \oplus T \mathcal{Z}(\mathfrak{g})$ is a maximal ideal of $\widetilde{\mathcal{Z}}(\mathfrak{g})$. Define the categories gr $\widetilde{\mathcal{C}}_{r}$ and $\operatorname{gr} \widetilde{\mathcal{C}} \infty$ for such $\widetilde{\chi} \in \operatorname{Max} \widetilde{\mathcal{Z}}(\mathfrak{g})$ in the same way as was done above for $\tilde{\chi} \in \operatorname{Max} \mathcal{Z}(\mathfrak{g})$. For fixed $\chi$, denote by $\mathcal{C}_{r}$ the category of non-graded $\mathfrak{g}_{0}$-modules $N$ satisfying $\chi^{r} N=0$ and by $\mathcal{C}_{\infty}$ the full subcategory of the category of non-graded $\mathfrak{g}_{0}$-modules consisting of the modules $N$ satisfying $N_{\chi}=\widetilde{N}$.

Under the above assumption on $\mathfrak{m}$, we prove in Section 4 the following

1.4.1. Theorem. The category $\operatorname{gr} \widetilde{\mathcal{C}}_{\infty}$ is equivalent to the category $\mathcal{C}_{\infty}$ where $\chi$ is a suitable maximal ideal of $\mathcal{Z}\left(\mathfrak{g}_{0}\right)$. The equivalence is given by the functors

$$
\widetilde{N} \mapsto \widetilde{N}_{\chi} \cap \widetilde{N}_{0}, \quad N \mapsto\left(\operatorname{Ind}_{\mathfrak{g}_{0}}^{\mathfrak{g}} N\right)_{\tilde{\chi}}
$$

where the grading on $\operatorname{Ind}_{\mathfrak{g}_{0}}^{\mathfrak{g}} N$ is determined by assuming $N$ to be even.

The restriction of these functors also provides the equivalence of gr $\widetilde{\mathcal{C}}_{r}$ with $\mathcal{C}_{r}$. 
A maximal ideal $\chi \in \operatorname{Max} \mathcal{Z}\left(\mathfrak{g}_{0}\right)$ is suitable in the sense of the above theorem iff for some projective Verma $\mathfrak{g}$-module $\widetilde{M}$ satisfying $\widetilde{\chi} \widetilde{M}=0$, the $\mathfrak{g}_{0}$-module $M:=$ $\widetilde{M}_{\chi} \cap \widetilde{M}_{0}$ is Verma and $\widetilde{M}=\widetilde{\mathcal{U}} M$. In 4.5.3 we construct such a suitable ideal $\chi$ for each $\mathfrak{m} \in \operatorname{Max} \mathcal{Z}(\mathfrak{g})$ satisfying the above assumption.

The proof is based on the theorem stating that the annihilator of a Verma $\mathfrak{g}$ module $\widetilde{M}$ satisfying $\mathfrak{m} \widetilde{M}=0$ is equal to $\widetilde{\mathcal{U}} \widetilde{\chi}$ (see [GL1], 6.2) and on Proposition4.7 which describes the locally finite part $F(\widetilde{M}, \widetilde{M})$ of endomorphisms of a Verma module $\widetilde{M}$ through the image of $\widetilde{\mathcal{U}}$ in it.

\section{Preliminaries}

Everywhere in the paper $\mathfrak{g}=\mathfrak{g}_{0} \oplus \mathfrak{g}_{1}$ denotes one (unless otherwise specified, an arbitrary one) of the basic classical complex Lie superalgebras $\mathfrak{g l}(m, n), \mathfrak{s l}(m, n)$, $\mathfrak{o} \mathfrak{s p}(m, n), \mathfrak{p s l}(n, n)$. Each of these Lie superalgebras possesses the following properties: it admits a $\mathfrak{g}$-invariant bilinear form which is non-degenerate on $[\mathfrak{g}, \mathfrak{g}]$ and the even part $\mathfrak{g}_{0}$ is a reductive Lie algebra.

2.1. Conventions. In this paper the ground field is $\mathbb{C}$. We denote by $\mathbb{N}^{+}$the set of positive integers. If $A$ is an algebra, $N$ is an $A$-module and $X, Y$ are subsets of $A$ and $N$ respectively, then we denote by $X Y$ the submodule spanned by the products $x y$ where $x \in X, y \in Y$.

For a $\mathbb{Z}_{2}$-homogeneous element $u$ of a superalgebra denote by $d(u)$ its $\mathbb{Z}_{2}$-degree. In all formulae where this notation is used, $u$ is assumed to be $\mathbb{Z}_{2}$-homogeneous.

For a Lie superalgebra $\mathfrak{m}$ denote by $\mathcal{U}(\mathfrak{m})$ its universal enveloping algebra and by $\mathcal{S}(\mathfrak{m})$ its symmetric algebra. All modules in the text are assumed to be left modules unless otherwise specified. An $\mathfrak{m}$-module $N$ is called locally finite if $\operatorname{dim} \mathcal{U}(\mathfrak{m}) v<\infty$ for all $v \in N$. Set $\widetilde{\mathcal{U}}:=\mathcal{U}(\mathfrak{g})$ and $\mathcal{U}:=\mathcal{U}\left(\mathfrak{g}_{0}\right)$.

The symbol $\widetilde{V}$ (resp., $V$ ) is always used for a simple $\mathfrak{g}$ - (resp., $\mathfrak{g}_{0^{-}}$)module and the symbol $\widetilde{M}$ (resp., $M$ ) for a Verma $\mathfrak{g}$ - (resp., $\left.\mathfrak{g}_{0^{-}}\right)$module.

2.2. Fix a triangular decomposition $\mathfrak{g}=\mathfrak{n}^{-} \oplus \mathfrak{h} \oplus \mathfrak{n}^{+}$- see [PS2] for a definition. Denote by $\Delta$ the set of all non-zero roots of $\mathfrak{g}$ and by $\Delta_{0}^{+}$(resp., $\Delta_{1}^{+}$) the set of non-zero positive even (resp., odd) roots of $\mathfrak{g}$. Set $\bar{\Delta}_{1}^{+}:=\left\{\beta \in \Delta_{1}^{+} \mid 2 \beta \notin \Delta_{0}^{+}\right\}$and

$$
\rho:=\frac{1}{2}\left(\sum_{\alpha \in \Delta_{0}^{+}} \alpha-\sum_{\beta \in \Delta_{1}^{+}} \beta\right) .
$$

Let $W$ be the Weyl group of $\mathfrak{g}_{0}$. For $w \in W, \mu \in \mathfrak{h}^{*}$ set

$$
w \cdot \mu=w(\mu+\rho)-\rho .
$$

Denote by $(-,-)$ a $\mathfrak{g}$-invariant bilinear form on $\mathfrak{g}$ which is non-degenerate on $[\mathfrak{g}, \mathfrak{g}]$ and also the induced $W$-invariant bilinear form on $\mathfrak{h}^{*}$. One has $\bar{\Delta}_{1}^{+}=\{\beta \in$ $\left.\Delta_{1}^{+} \mid(\beta, \beta)=0\right\}$.

2.3. Define the adjoint action of $\mathfrak{g}$ on $\widetilde{\mathcal{U}}$ by setting

$$
(\operatorname{ad} g) u=g u-(-1)^{d(g) d(u)} u g, \forall g \in \mathfrak{g}, u \in \widetilde{\mathcal{U}} .
$$

By default, the action of $\mathfrak{g}$ on $\widetilde{\mathcal{U}}$ is assumed to be the adjoint action. The centre $\mathcal{Z}(\mathfrak{g})$ of $\widetilde{\mathcal{U}}$ is equal to $\widetilde{\mathcal{U}}^{\text {ad } \mathfrak{g}}$. 
Define the twisted adjoint action of $\mathfrak{g}$ on $\widetilde{\mathcal{U}}$ by setting

$$
\left(\operatorname{ad}^{\prime} g\right) u=g u-(-1)^{d(g)(d(u)+1)} u g, \forall g \in \mathfrak{g}, u \in \widetilde{\mathcal{U}} .
$$

The anticentre $\mathcal{A}(\mathfrak{g}):=\widetilde{\mathcal{U}}^{\mathrm{ad}^{\prime} \mathfrak{g}}$ contains an element $T$ defined in [G1]. This is a unique element of $\mathcal{A}(\mathfrak{g})$ satisfying $\mathcal{P}(T)(\lambda)=\prod_{\beta \in \Delta_{1}^{+}}(\beta, \lambda+\rho)$ for any $\lambda \in \mathfrak{h}^{*}$; here $\mathcal{P}: \widetilde{\mathcal{U}} \rightarrow \mathcal{S}(\mathfrak{h})$ is the Harish-Chandra projection. The element $T$ is even. Since $T$ belongs to $\mathcal{A}(\mathfrak{g})$, it commutes with the even elements of $\widetilde{\mathcal{U}}$ and anticommutes with the odd ones; in particular, $T^{2} \in \mathcal{Z}(\mathfrak{g})$.

The Harish-Chandra projection $\mathcal{P}$ provides a monomorphism $\iota: \mathcal{Z}(\mathfrak{g}) \rightarrow \mathcal{S}(\mathfrak{h})^{W}$. The image of $\iota$ is described in [K2], [S1, [BZV]; $\iota$ is bijective iff $\mathfrak{g}=\mathfrak{o s p}(1,2 l)$. The centre $\mathcal{Z}(\mathfrak{g})$ contains an element $Q$ such that

$$
\mathcal{P}(Q)(\lambda)=\prod_{\beta \in \bar{\Delta}_{1}^{+}}(\beta, \lambda+\rho) .
$$

The localized algebra $\mathcal{Z}(\mathfrak{g})\left[Q^{-1}\right]$ is isomorphic to a localization of a polynomial algebra $\mathcal{S}(\mathfrak{h})^{W}$ by $\mathcal{P}(Q)$ - see [K3]. The localized algebra $\mathcal{Z}(\mathfrak{g})\left[T^{-2}\right]$ is isomorphic to a localization of a polynomial algebra $\mathcal{S}(\mathfrak{h})^{W}$.

2.4. The category $\widetilde{\mathcal{O}}$ and Verma modules. Denote by $\mathcal{O}$ the full subcategory of the category of $\mathfrak{g}_{0}$-modules consisting of finitely generated $\mathfrak{h}$-diagonalizable $\mathfrak{g}_{0^{-}}$ modules which are $\mathfrak{n}_{0}^{+}$-locally finite. Denote by $\widetilde{\mathcal{O}}$ the similarly defined category of $\mathfrak{g}$-modules. Since $\mathcal{U}\left(\mathfrak{n}^{+}\right)$is finite over $\mathcal{U}\left(\mathfrak{n}_{0}^{+}\right)$, a $\mathfrak{g}$-module $N$ belongs to $\widetilde{\mathcal{O}}$ iff as a $\mathfrak{g}_{0}$-module $N$ belongs to $\mathcal{O}$. In particular, any module of category $\widetilde{\mathcal{O}}$ has a finite length.

2.4.1. For $\lambda \in \mathfrak{h}^{*}$ denote by $\mathbb{C}_{\lambda}$ a one-dimensional $\mathfrak{b}$-module such that $\mathfrak{n}^{+} v=0$ and $h v=\lambda(h) v$ for any $h \in \mathfrak{h}, v \in \mathbb{C}_{\lambda}$. Define a Verma module $\widetilde{M}(\lambda)$ by setting

$$
\widetilde{M}(\lambda):=\widetilde{\mathcal{U}} \otimes_{\mathcal{U}(\mathfrak{b})} \mathbb{C}_{\lambda} .
$$

A Verma $\mathfrak{g}_{0}$-module $M(\lambda)$ is defined similarly.

2.4.2. A maximal ideal $\tilde{\chi} \in \operatorname{Max} \mathcal{Z}(\mathfrak{g})$ is called typical if it does not contain $Q$ defined in [2.3. If $\tilde{\chi} \in \operatorname{Max} \mathcal{Z}(\mathfrak{g})$ is typical, then the set

$$
W(\widetilde{\chi}):=\left\{\lambda \in \mathfrak{h}^{*} \mid \widetilde{\chi} \widetilde{M}(\lambda)=0\right\}
$$

forms a single $W$-orbit. For $\mathfrak{g}=\mathfrak{o s p}(1,2 l)$ all $\tilde{\chi} \in \operatorname{Max} \mathcal{Z}(\mathfrak{g})$ are typical.

Call a $\mathfrak{g}$-module $N$ typical if $\operatorname{Ann}_{\mathcal{Z}(\mathfrak{g})} N$ is a typical maximal ideal of $\mathcal{Z}(\mathfrak{g})$. Define the partial order on $\mathfrak{h}^{*}$ by setting $\mu \geq \nu \Longleftrightarrow(\mu-\nu) \in \sum_{\alpha \in \Delta^{+}} \mathbb{N} \alpha$. A typical Verma module $\widetilde{M}(\lambda)$ is projective (resp., simple) in $\widetilde{\mathcal{O}}$ if $\lambda$ is maximal (resp., minimal) in $W . \lambda$-see, for instance, G2, 2.5.3. In particular, for a typical $\tilde{\chi} \in \operatorname{Max} \mathcal{Z}(\mathfrak{g})$ there exists a projective Verma module $\widetilde{M}$ satisfying $\widetilde{\chi} \widetilde{M}=0$.

A maximal ideal $\tilde{\chi} \in \operatorname{Max} \mathcal{Z}(\mathfrak{g})$ is called strongly typical if $T^{2} \notin \widetilde{\chi}$. A strongly typical central character is typical. Call a $\mathfrak{g}$-module $N$ strongly typical if $\operatorname{Ann}_{\mathcal{Z}(\mathfrak{g})} N$ is a strongly typical maximal ideal of $\mathcal{Z}(\mathfrak{g})$.

If $\mathfrak{g}$ is not of types $B(m, n), G(3)$, then $\Delta_{1}^{+}=\bar{\Delta}_{1}^{+}$and so the notion of typical and strongly typical modules coincide. 
2.5. Throughout the paper we shall write "ad $\mathfrak{g}$-module" instead of " $\mathfrak{g}$-module with respect to the adjoint action". For any $\mathfrak{g}$-modules $N_{1}, N_{2}$ view $\operatorname{Hom}\left(N_{1}, N_{2}\right):=$ $\operatorname{Hom}_{\mathbb{C}}\left(N_{1}, N_{2}\right)$ as a $\mathfrak{g}$-module with respect to the adjoint action and denote by $F\left(N_{1}, N_{2}\right)$ the locally finite part of the ad $\mathfrak{g}$-module $\operatorname{Hom}\left(N_{1}, N_{2}\right)$. Similarly for any $\mathfrak{g}_{0}$-modules $N_{1}, N_{2}$ denote by $F\left(N_{1}, N_{2}\right)$ the locally finite part of the ad $\mathfrak{g}_{0}$-module $\operatorname{Hom}\left(N_{1}, N_{2}\right)$. Notice that for any $\mathfrak{g}$-module $N$ its locally finite part coincides with its ad $\mathfrak{g}_{0}$-locally finite part, since $\tilde{\mathcal{U}}$ is a finite extension of $\mathcal{U}$.

2.5.1. Let $N$ be a $\mathfrak{g}_{0}$-module, and let $E$ be a finite-dimensional $\mathfrak{g}_{0}$-module. It is easy to check that $A:=F(N, N)$ and $B:=F(N \otimes E, N \otimes E)$ are subalgebras of $\operatorname{Hom}(N, N)$ and $\operatorname{Hom}(N \otimes E, N \otimes E)$, respectively. The algebra $A$ acts on $F(N, N \otimes E)$ from the right and the algebra $B$ acts on $F(N, N \otimes E)$ from the left; these actions commute. We claim that $F(N, N \otimes E)$ is a free right $A$-module whose rank is equal to the dimension of $E$ and, moreover, $\operatorname{End}_{A}(F(N, N \otimes E))=B$.

Indeed, consider the map $\iota: F(N, N) \otimes E \rightarrow \operatorname{Hom}(N, N \otimes E)$ given by $\iota(f \otimes v)(n)=f(n) \otimes v$ for any $f \in F(N, N), v \in E$, and the map $\iota^{\prime}: F(N, N \otimes E) \rightarrow$ $\operatorname{Hom}(N, N) \otimes E$ given by $\psi \mapsto \sum_{i} p_{i} \circ \psi \otimes e_{i}$ where $\left\{e_{i}\right\}$ is a basis of $E$ and $p_{i}: N \otimes E \rightarrow N$ are given by $p_{i}\left(n \otimes e_{j}\right):=\delta_{i, j} n \otimes e_{j}$. One can easily see that $\operatorname{Im} \iota \subseteq F(N, N \otimes E), \operatorname{Im} \iota^{\prime} \subseteq F(N, N) \otimes E$ and that $\iota^{\prime} \circ \iota=\mathrm{id}, \iota \circ \iota^{\prime}=\mathrm{id}$. Thus $F(N, N \otimes E) \cong F(N, N) \otimes E$ is a free right $A$-module whose rank is equal to the dimension of $E$. Similarly, the map $\iota^{\prime \prime}: A \otimes \operatorname{End}_{\mathbb{C}}(E) \rightarrow B$ given by $\iota^{\prime \prime}(f \otimes \phi)(n \otimes v):=$ $f(n) \otimes \phi(v)$ for any $f \in A=F(N, N), n \in N, v \in E, \phi \in \operatorname{End}_{\mathbb{C}}(E)$ is bijective. For any $f, f^{\prime} \in A, v \in E, \phi \in \operatorname{End}_{\mathbb{C}}(E)$ one has $\iota^{\prime \prime}(f \otimes \phi)\left(\iota\left(f^{\prime} \otimes v\right)\right)=\iota\left(f f^{\prime} \otimes \phi(v)\right)$. This implies $\operatorname{End}_{A}(F(N, N \otimes E))=B$ since the $A$-module $F(N, N \otimes E)$ is freely generated by the elements $\iota\left(1 \otimes e_{i}\right)$ (here 1 is the unit of $A$ ).

2.6. Let $M$ be a Verma $\mathfrak{g}$-module. By Duflo's theorem Ann $M=\mathcal{U} \operatorname{Ann}_{\mathcal{Z}\left(\mathfrak{g}_{0}\right)} M-$ see [D]. By [J1], 6.4, the natural map $\mathcal{U} /(\operatorname{Ann} M) \rightarrow F(M, M)$ is bijective.

Let $\widetilde{M}$ be a strongly typical Verma $\mathfrak{g}$-module. Then $\operatorname{Ann} \widetilde{M}=\widetilde{\mathcal{U}} \operatorname{Ann}_{\mathcal{Z}(\mathfrak{g})} \widetilde{M}$ and the natural map $\widetilde{\mathcal{U}} /(\operatorname{Ann} \widetilde{M}) \rightarrow F(\widetilde{M}, \widetilde{M})$ is bijective (see [G2], 9.4,9.5).

2.7. For a $\mathfrak{g}$-module $\widetilde{N}$ and a maximal ideal $\tilde{\chi} \in \mathcal{Z}(\mathfrak{g})$ set

$$
\widetilde{N}_{\tilde{\chi}}:=\left\{v \in \widetilde{N} \mid \widetilde{\chi}^{r} v=0, r>>0\right\} .
$$

We say that a $\widetilde{\mathcal{U}}$-module $\widetilde{N}$ has a finite support $\operatorname{supp}_{\mathcal{Z}(\mathfrak{g})} \tilde{N}=\left\{\widetilde{\chi}_{1}, \ldots, \widetilde{\chi}_{k}\right\}$ if for any $v \in \widetilde{N}$ there exist $r_{1}, \ldots, r_{k} \in \mathbb{N}^{+}$such that $\prod_{i} \widetilde{\chi}_{i}^{r_{i}} v=0$. In this case,

$$
\widetilde{N}=\bigoplus_{i} \tilde{N}_{\tilde{\chi}_{i}}
$$

and each $\widetilde{N}_{\widetilde{\chi}_{i}}$ is canonically isomorphic to the localization of the module $\widetilde{N}$ at $\widetilde{\chi}_{i}$. If $\widetilde{N}$ has a finite support and $0 \rightarrow \widetilde{N}^{\prime} \rightarrow \widetilde{N} \rightarrow \widetilde{N}^{\prime \prime} \rightarrow 0$ is an exact sequence, then, for any $\widetilde{\chi}^{\prime} \in \operatorname{Max} \mathcal{Z}(\mathfrak{g})$, the sequence $0 \rightarrow \widetilde{N}_{\widetilde{\chi}^{\prime}}^{\prime} \rightarrow \widetilde{N}_{\widetilde{\chi}^{\prime}} \rightarrow \widetilde{N}_{\widetilde{\chi}^{\prime}}^{\prime \prime} \rightarrow 0$ is also exact.

We adopt similar notation for $\mathcal{Z}\left(\mathfrak{g}_{0}\right)$ and $\mathcal{U}$-modules. For a graded $\mathfrak{g}$-module $\widetilde{N}$ and a maximal ideal $\chi$ of $\mathcal{Z}\left(\mathfrak{g}_{0}\right)$ we set

$$
\widetilde{N}_{\chi ; i}:=\widetilde{N}_{\chi} \cap \widetilde{N}_{i}
$$

for $i=0,1$. 
2.7.1. Let $\widetilde{\chi}$ be a strongly typical central character, and let $\widetilde{M}$ be such that $\widetilde{\chi} \widetilde{M}=$ 0 . Then

$$
\widetilde{\mathcal{U}} \widetilde{\chi} \cap \mathcal{Z}\left(\mathfrak{g}_{0}\right)=\operatorname{Ann} \widetilde{M} \cap \mathcal{Z}\left(\mathfrak{g}_{0}\right)=\prod_{\chi \in \operatorname{supp}_{\mathcal{Z}\left(\mathfrak{g}_{0}\right)} \widetilde{M}} \chi^{r(\chi)}
$$

where $r(\chi)$ is the minimal $r$ such that $\chi^{r} \widetilde{M}_{\chi}=0$. In particular, if $\chi \in \operatorname{Max} \mathcal{Z}\left(\mathfrak{g}_{0}\right)$ is such that $\widetilde{M}_{\chi}$ is a Verma $\mathfrak{g}_{0}$-module, then $r(\chi)=1$. Moreover, any $\mathfrak{g}$-module $\widetilde{N}$ satisfying $\tilde{\chi} \widetilde{N}=0$ has a finite support in $\mathcal{Z}\left(\mathfrak{g}_{0}\right)$ (which is a subset of $\left.\operatorname{supp}_{\mathcal{Z}\left(\mathfrak{g}_{0}\right)} \widetilde{M}\right)$ and one has $\chi \widetilde{N}_{\chi}=0$ if $\widetilde{M}_{\chi}$ is a Verma $\mathfrak{g}_{0}$-module.

2.7.2. For a strongly typical $\tilde{\chi} \in \operatorname{Max} \mathcal{Z}(\mathfrak{g})$ call $\chi \in \operatorname{Max} \mathcal{Z}\left(\mathfrak{g}_{0}\right)$ a perfect mate if

(i) For any Verma $\mathfrak{g}$-module $\widetilde{M}$ annihilated by $\widetilde{\chi}$, the $\mathfrak{g}_{0}$-module $\widetilde{M}_{\chi}$ is Verma.

(ii) For any non-trivial $\mathfrak{g}$-module $\widetilde{N}$ annihilated by $\widetilde{\chi}$, the $\mathfrak{g}_{0}$-module $\widetilde{N}_{\chi}$ is nontrivial.

In G2, Section 8, we describe a perfect mate for each strongly typical $\tilde{\chi} \in$ $\operatorname{Max} \mathcal{Z}(\mathfrak{g})$.

2.8. For a graded $\mathfrak{g}_{0}$-module $L$ denote by $\operatorname{Ind}_{\mathfrak{g}_{0}}^{\mathfrak{g}} L$ the vector space $\widetilde{\mathcal{U}} \otimes \mathcal{U} L$ (here $\widetilde{\mathcal{U}}$ is considered as a right $\mathcal{U}$-module and a left $\widetilde{\mathcal{U}}$-module through the multiplication) equipped with the natural structure of a left graded $\widetilde{\mathcal{U}}$-module. Denote by Coind $\mathfrak{g}_{\mathfrak{g}_{0}}^{\mathfrak{g}} L$ the vector space $\operatorname{Hom}_{\mathcal{U}}(\widetilde{\mathcal{U}}, L)$ (here $\widetilde{\mathcal{U}}$ is considered as a left $\mathcal{U}$-module) equipped with the following structure of a left graded $\widetilde{\mathcal{U}}$-module: $(u f)\left(u^{\prime}\right):=f\left(u^{\prime} u\right)$ for any $f \in \operatorname{Hom}_{\mathcal{U}}(\widetilde{\mathcal{U}}, L), u, u^{\prime} \in \widetilde{\mathcal{U}}$. For a graded $\mathfrak{g}$-module $\widetilde{N}$ and a graded $\mathfrak{g}_{0}$-module $L$ one has the canonical bijections

$$
\begin{gathered}
\operatorname{Hom}_{\mathfrak{g}_{0}}(\tilde{N}, L) \stackrel{\sim}{\longrightarrow} \operatorname{Hom}_{\mathfrak{g}}\left(\tilde{N}, \operatorname{Coind}_{\mathfrak{g}_{0}}^{\mathfrak{g}} L\right), \\
\operatorname{Hom}_{\mathfrak{g}_{0}}(L, \widetilde{N}) \stackrel{\sim}{\longrightarrow} \operatorname{Hom}_{\mathfrak{g}}\left(\operatorname{Ind}_{\mathfrak{g}_{0}}^{\mathfrak{g}} L, \widetilde{N}\right) .
\end{gathered}
$$

By $\left[\mathrm{BF}, \operatorname{Ind}_{\mathfrak{g}_{0}}^{\mathfrak{g}} L \cong \operatorname{Coind}_{\mathfrak{g}_{0}}^{\mathfrak{g}} L\right.$ for any graded $\mathfrak{g}_{0}$-module $L$.

The same formulae define non-graded versions of $\operatorname{Ind}_{\mathfrak{g}_{0}}^{\mathfrak{g}} L$ and $\operatorname{Coind}_{\mathfrak{g}_{0}}^{\mathfrak{g}} L$. The same canonical bijections (11) take place.

\section{EquivalenCE OF CATEgORIES}

\section{FOR A STRONGLY TYPICAL CENTRAL CHARACTER}

In this section we prove that the category of $\widetilde{\mathcal{U}}$-modules $\widetilde{N}$ satisfying $\widetilde{N}=\widetilde{N}_{\tilde{\chi}}$ is equivalent to the category of $\mathcal{U}$-modules $N$ satisfying $N=N_{\chi}$ provided $\tilde{\chi} \in$ $\operatorname{Max} \mathcal{Z}(\mathfrak{g})$ is strongly typical and $\chi \in \operatorname{Max} \mathcal{Z}\left(\mathfrak{g}_{0}\right)$ is its perfect mate see 2.7.2. The corresponding quasi-inverse functors are given by $\widetilde{N} \mapsto \widetilde{N}_{\chi}$ and by $N \mapsto\left(\operatorname{Ind}_{\mathfrak{g}_{0}}^{\mathfrak{g}} N\right)_{\tilde{\chi}}$. There are two versions of this result: graded and non-graded. We consider first a non-graded version. A graded version is easily deduced from the non-graded one.

3.1. Notation. Take a strongly typical $\tilde{\chi} \in \operatorname{Max} \mathcal{Z}(\mathfrak{g})$ and let $\chi \in \operatorname{Max} \mathcal{Z}\left(\mathfrak{g}_{0}\right)$. Denote by $\widetilde{\mathcal{C}}_{r}\left(r \in \mathbb{N}^{+}\right)$the category of non-graded $\widetilde{\mathcal{U}}$-modules $\widetilde{N}$ satisfying $\tilde{\chi}^{r} \widetilde{N}=0$ and by $\widetilde{\mathcal{C}}_{\infty}$ the category of non-graded $\widetilde{\mathcal{U}}$-modules $\widetilde{N}$ satisfying $\widetilde{N}=\widetilde{N}_{\tilde{\chi}}$. Similarly, let $\mathcal{C}_{r}$ be the category of $\mathcal{U}$-modules $N$ satisfying $\chi^{r} N=0$, and let $\mathcal{C}_{\infty}$ be the category of $\mathcal{U}$-modules $N$ satisfying $N=N_{\chi}$. Evidently $\widetilde{\mathcal{C}}_{r}$ is a full subcategory of $\widetilde{\mathcal{C}}_{r+1}$ and any module in $\widetilde{\mathcal{C}}_{\infty}$ is a direct limit of modules belonging to $\widetilde{\mathcal{C}}_{r}$ for $r \rightarrow \infty$. 
3.1.1. Let $M$ be a Verma $\mathfrak{g}_{0}$-module such that $\chi M=0$. We shall use the equality

$$
\operatorname{Ann}_{\tilde{\mathcal{U}}}\left(\operatorname{Ind}_{\mathfrak{g}_{0}}^{\mathfrak{g}} M\right)=\widetilde{\mathcal{U}} \chi
$$

which follows from the fact that $\widetilde{\mathcal{U}}$ is free over $\mathcal{U}$.

Let us show that

$$
\operatorname{supp}_{\mathcal{Z}(\mathfrak{g})} \operatorname{Ind}_{\mathfrak{g}_{0}}^{\mathfrak{g}} N \subseteq \operatorname{supp}_{\mathcal{Z}(\mathfrak{g})} \operatorname{Ind}_{\mathfrak{g}_{0}}^{\mathfrak{g}} M
$$

for any $N \in \mathcal{C}_{\infty}$. Indeed, for any $N \in \mathcal{C}_{1}$

$$
\operatorname{Ann}_{\mathcal{Z}(\mathfrak{g})}\left(\operatorname{Ind}_{\mathfrak{g}_{0}}^{\mathfrak{g}} N\right) \supseteq \widetilde{\mathcal{U}} \chi \cap \mathcal{Z}(\mathfrak{g})=\operatorname{Ann}_{\mathcal{Z}(\mathfrak{g})}\left(\operatorname{Ind}_{\mathfrak{g}_{0}}^{\mathfrak{g}} M\right)
$$

which implies the inclusion (2) (for $N \in \mathcal{C}_{1}$ ). Any $N \in \mathcal{C}_{r}$ admits a finite filtration with the factors belonging to $\mathcal{C}_{1}$ and so the inclusion (2) holds for such $N$. To deduce (2) for any $N \in \mathcal{C}_{\infty}$, observe that for any $v \in \operatorname{Ind}_{\mathfrak{g}_{0}}^{\mathfrak{g}} N$ there exists a finitely generated submodule $N^{\prime}$ of $N$ such that $\operatorname{Ind}_{\mathfrak{g}_{0}}^{\mathfrak{g}} N^{\prime}$ contains $v$. Since $N^{\prime}$ is finitely generated, it lies in $\mathcal{C}_{r}$ for a suitable $r \in \mathbb{N}^{+}$. This implies the inclusion (2).

Hence for any $N \in \mathcal{C}_{\infty}$

$$
\operatorname{Ind}_{\mathfrak{g}_{0}}^{\mathfrak{g}} N=\bigoplus_{\tilde{\chi}^{\prime} \in \operatorname{supp}_{\mathcal{Z}(\mathfrak{g})} \operatorname{Ind}_{\mathfrak{g}_{0}}^{\mathfrak{g}} M}\left(\operatorname{Ind}_{\mathfrak{g}_{0}}^{\mathfrak{g}} N\right)_{\tilde{\chi}^{\prime}}
$$

Similarly, choose $\widetilde{M}$ such that $\tilde{\mathcal{U}} \widetilde{\chi}=\operatorname{Ann} \widetilde{M}$. Then for any $\widetilde{N} \in \widetilde{\mathcal{C}_{\infty}}$

$$
\widetilde{N}=\bigoplus_{\chi^{\prime} \in \operatorname{supp}_{\mathcal{Z}\left(\mathfrak{g}_{0}\right)} \widetilde{M}} \widetilde{N}_{\chi^{\prime}}
$$

3.1.2. Consider the map $f: \widetilde{\mathcal{U}} /(\widetilde{\mathcal{U}} \chi) \rightarrow F\left(M, \operatorname{Ind}_{\mathfrak{g}_{0}}^{\mathfrak{g}} M\right)$ induced by the natural map $\widetilde{\mathcal{U}} \rightarrow F\left(M, \operatorname{Ind}_{\mathfrak{g}_{0}}^{\mathfrak{g}} M\right)$ given by $u \mapsto(m \mapsto u \otimes m)$. We claim $f$ is an isomorphism of left $\widetilde{\mathcal{U}}$ - and right $\mathcal{U}$-modules. Since $\widetilde{\mathcal{U}}$ is free over $\mathcal{U}$ and $\operatorname{Ann}_{\mathcal{U}} M=\mathcal{U} \chi$, the map $f$ is injective. Kostant's Separation Theorem (see [Ko]) states the existence of an ad $\mathfrak{g}_{0}$-submodule $H^{\prime}$ of $\mathcal{S}\left(\mathfrak{g}_{0}\right)$ such that the multiplication map provides an isomorphism $H^{\prime} \otimes \mathcal{S}\left(\mathfrak{g}_{0}\right)^{\mathfrak{g}_{0}} \stackrel{\sim}{\longrightarrow} \mathcal{S}\left(\mathfrak{g}_{0}\right)$. Then

$$
\mathcal{S}(\mathfrak{g})=\Lambda \mathfrak{g}_{1} \otimes \mathcal{S}\left(\mathfrak{g}_{0}\right)=\left(\Lambda \mathfrak{g}_{1} \otimes H^{\prime}\right) \otimes \mathcal{S}\left(\mathfrak{g}_{0}\right)^{\mathfrak{g}_{0}} .
$$

Using $\left[\mathrm{BL}, 5.4\right.$, it is easy to deduce the existence of an ad $\mathfrak{g}_{0}$-submodule $H$ of $\widetilde{\mathcal{U}}$ such that the multiplication map provides the isomorphism $H \otimes \mathcal{Z}\left(\mathfrak{g}_{0}\right) \rightarrow \widetilde{\mathcal{U}}$. Moreover, $H \cong \Lambda \mathfrak{g}_{1} \otimes H^{\prime}$ as an ad $\mathfrak{g}_{0}$-module. By [2.6] $H^{\prime} \cong F(M, M)$ as ad $\mathfrak{g}_{0^{-}}$ modules. The chain of ad $\mathfrak{g}_{0}$-isomorphisms

$$
\widetilde{\mathcal{U}} /(\widetilde{\mathcal{U}} \chi) \cong \Lambda \mathfrak{g}_{1} \otimes H^{\prime} \cong \Lambda \mathfrak{g}_{1} \otimes F(M, M) \cong F\left(M, \operatorname{Ind}_{\mathfrak{g}_{0}}^{\mathfrak{g}} M\right)
$$

implies the surjectivity of $f$.

The bijectivity of $f$ gives the following useful formula for any $N \in \mathcal{C}_{1}$ :

$$
\operatorname{Ind}_{\mathfrak{g}_{0}}^{\mathfrak{g}} N=F\left(M, \operatorname{Ind}_{\mathfrak{g}_{0}}^{\mathfrak{g}} M\right) \otimes \mathcal{U} N
$$

3.2. Fix any $\chi \in \operatorname{Max} \mathcal{Z}\left(\mathfrak{g}_{0}\right), \tilde{\chi} \in \operatorname{Max} \mathcal{Z}(\mathfrak{g})$. Define the functors $\Psi: \widetilde{\mathcal{C}}_{\infty} \rightarrow \mathcal{C}_{\infty}$ and $\Phi: \mathcal{C}_{\infty} \rightarrow \widetilde{\mathcal{C}}_{\infty}$ by the formulae

$$
\Psi(\widetilde{N})=\widetilde{N}_{\chi}, \quad \Phi(N)=\left(\operatorname{Ind}_{\mathfrak{g}_{0}}^{\mathfrak{g}} N\right)_{\tilde{\chi}}
$$

3.2.1. Lemma. The functor $\Psi$ is left and right adjoint to $\Phi$. 
Proof. Using (3), one obtains

$$
\begin{aligned}
\operatorname{Hom}_{\mathfrak{g}}(\Phi(N), \widetilde{N}) & =\operatorname{Hom}_{\mathfrak{g}}\left(\left(\operatorname{Ind}_{\mathfrak{g}_{0}}^{\mathfrak{g}} N\right) \tilde{\chi}, \widetilde{N}\right) \\
& =\operatorname{Hom}_{\mathfrak{g}}\left(\operatorname{Ind}_{\mathfrak{g}_{0}}^{\mathfrak{g}} N, \widetilde{N}\right)=\operatorname{Hom}_{\mathfrak{g}_{0}}(N, \widetilde{N}) \\
& =\operatorname{Hom}_{\mathfrak{g}_{0}}\left(N, \widetilde{N}_{\chi}\right)=\operatorname{Hom}_{\mathfrak{g}_{0}}(N, \Psi(\widetilde{N}))
\end{aligned}
$$

for any $N \in \mathcal{C}_{\infty}, \widetilde{N} \in \widetilde{\mathcal{C}}_{\infty}$, and also, using (4),

$$
\begin{aligned}
\operatorname{Hom}_{\mathfrak{g}}(\widetilde{N}, \Phi(N)) & =\operatorname{Hom}_{\mathfrak{g}}\left(\widetilde{N},\left(\operatorname{Ind}_{\mathfrak{g}_{0}}^{\mathfrak{g}} N\right)_{\tilde{\chi}}\right) \\
& =\operatorname{Hom}_{\mathfrak{g}}\left(\widetilde{N}, \operatorname{Ind}_{\mathfrak{g}_{0}}^{\mathfrak{g}} N\right) \stackrel{\sim}{\longrightarrow} \operatorname{Hom}_{\mathfrak{g}}\left(\widetilde{N}, \operatorname{Coind}_{\mathfrak{g}_{0}}^{\mathfrak{g}} N\right) \\
& =\operatorname{Hom}_{\mathfrak{g}_{0}}(\widetilde{N}, N)=\operatorname{Hom}_{\mathfrak{g}_{0}}\left(\widetilde{N}_{\chi}, N\right)=\operatorname{Hom}_{\mathfrak{g}_{0}}(\Psi(\widetilde{N}), N)
\end{aligned}
$$

where $=$ stands for the natural isomorphisms and $\stackrel{\sim}{\longrightarrow}$ is induced by an isomorphism $\operatorname{Ind}_{\mathfrak{g}_{0}}^{\mathfrak{g}} N \cong \operatorname{Coind}_{\mathfrak{g}_{0}}^{\mathfrak{g}} N$ (see 2.8) .

3.3. Conventions. Fix a strongly typical $\tilde{\chi} \in \operatorname{Max} \mathcal{Z}(\mathfrak{g})$ and a Verma $\mathfrak{g}$-module $\widetilde{M}$ which is projective in $\widetilde{\mathcal{O}}$ and such that $\widetilde{\chi} \widetilde{M}=0$. Take $\chi \in \operatorname{Max} \mathcal{Z}\left(\mathfrak{g}_{0}\right)$ such that

$$
\begin{aligned}
& \text { (a) } M:=\widetilde{M}_{\chi} \text { is a Verma } \mathfrak{g}_{0} \text {-module, } \\
& \text { (b) } \widetilde{M}=\widetilde{\mathcal{U}} M .
\end{aligned}
$$

For instance, one can choose $\chi$ to be a perfect mate for $\tilde{\chi}$ (see 2.7.2).

Until the end of this section $\widetilde{\chi}, \widetilde{M}$ and $\chi$ chosen as above are assumed to be fixed.

\subsubsection{Theorem. The functors}

$$
\begin{aligned}
\Psi: \widetilde{\mathcal{C}}_{\infty} & \rightarrow \mathcal{C}_{\infty}, \quad \widetilde{N} \mapsto \widetilde{N}_{\chi}, \\
\Phi: \mathcal{C}_{\infty} \rightarrow \widetilde{\mathcal{C}}_{\infty}, & N \mapsto\left(\operatorname{Ind}_{\mathfrak{g}_{0}}^{\mathfrak{g}} N\right)_{\tilde{\chi}}
\end{aligned}
$$

are mutually quasi-inverse. Moreover, their restrictions provide the equivalence of the categories $\widetilde{\mathcal{C}}_{r}$ and $\mathcal{C}_{r}$ for any $r \in \mathbb{N}^{+}$.

Outline of the proof. We know by 3.2.1 that the functors $\Psi, \Phi$ are adjoint. In 3.4.1 we reduce the required assertion to the "case $r=1$ ", that is, to the statement that the restrictions $\Psi_{1}, \Phi_{1}$ of the functors $\Psi, \Phi$ to the categories $\widetilde{\mathcal{C}}_{1}, \mathcal{C}_{1}$ provide an equivalence of the categories. Note that 3.4.1 does not use condition (6).

Observe that the inclusion $\Psi_{1}\left(\widetilde{\mathcal{C}}_{1}\right) \subseteq \mathcal{C}_{1}$ immediately follows from [2.7.1, but it is not clear apriori that $\Phi_{1}\left(\mathcal{C}_{1}\right) \subseteq \widetilde{\mathcal{C}}_{1}$. To prove that $\Psi_{1}, \Phi_{1}$ provide an equivalence of the categories we show, using Proposition 3.4.3, that the functor $\Phi^{\prime}: N \mapsto$ $F(M, \widetilde{M}) \otimes \mathcal{U} N$ provides an equivalence of the categories $\mathcal{C}_{1} \rightarrow \widetilde{\mathcal{C}}_{1}$. It is easy to show that $\Psi_{1}$ is the left quasi-inverse to $\Phi^{\prime}$ and so it provides an equivalence of the categories $\widetilde{\mathcal{C}}_{1} \rightarrow \mathcal{C}_{1}$. Using this fact, we show that $\Phi(M) \cong \widetilde{M}$ and deduce that $\Phi_{1}$ is isomorphic to $\Phi^{\prime}$. This will complete the proof.

\subsection{Proof of Theorem 3.3.1,}

3.4.1. Reduction to the case $r=1$. Formula (3) implies that $\Phi(N)$ is isomorphic to the localization of $\operatorname{Ind}_{\mathfrak{g}_{0}}^{\mathfrak{g}} N$, considered as a $\mathcal{Z}(\mathfrak{g})$-module, at the maximal ideal $\tilde{\chi}$ (that is, by the set $\mathcal{Z}(\mathfrak{g}) \backslash \widetilde{\chi})$.

Similarly, formula (4) implies that $\Psi(\widetilde{N})$ is isomorphic to the localization of $\widetilde{N}$, considered as a $\mathcal{Z}\left(\mathfrak{g}_{0}\right)$-module, at the maximal ideal $\chi$. 
Taking into account that the induction and the localization functors are exact and commute with direct limits, one concludes that the assertion of Theorem 3.3 .1 is equivalent to the statement that the restrictions $\Psi_{1}, \Phi_{1}$ of the functors $\Psi$, $\Phi$ to the categories $\widetilde{\mathcal{C}}_{1}, \mathcal{C}_{1}$ provide an equivalence of the categories.

3.4.2. Case $r=1$. It remains to show that the restriction $\Psi_{1}$ of $\Psi$ to the subcategory $\widetilde{\mathcal{C}}_{1}$ and the restriction $\Phi_{1}$ of $\Phi$ to the subcategory $\mathcal{C}_{1}$ provide an equivalence of the categories $\widetilde{\mathcal{C}}_{1}$ and $\mathcal{C}_{1}$.

We start with the following technical proposition.

3.4.3. Lemma. Assume that $\tilde{N}$ is a $\mathfrak{g}$-module, $N$ is a $\mathfrak{g}_{0}$-direct summand of $\tilde{N}$ and $\widetilde{N}$ is a $\mathfrak{g}$-direct summand of $\operatorname{Ind}_{\mathfrak{g}_{0}}^{\mathfrak{g}} N$. Then the $F(\widetilde{N}, \widetilde{N})-F(N, N)$ bimodule $F(N, \widetilde{N})$ provides a Morita equivalence between the algebras $F(N, N)$ and $F(\tilde{N}, \widetilde{N})$.

Proof. Denote the algebra $F(N, N)$ by $A$. For any $\mathfrak{g}$-module $X$ endow the vector space $F(N, X)$ with the natural right $A$-module structure. Since $N$ is a $\mathfrak{g}_{0}$-direct summand of $\widetilde{N}, A$ as a right module over itself is a direct summand of $F(N, \widetilde{N})$. To obtain the statement one has to check that $F(N, \widetilde{N})$ is a finitely generated projective right $A$-module and that $\operatorname{End}_{A}(F(N, \widetilde{N}))=F(\widetilde{N}, \widetilde{N})$.

Denote $\operatorname{Ind}_{\mathfrak{g}_{0}}^{\mathfrak{g}} N$ by $I$. Recall that as a $\mathfrak{g}_{0}$-module $I=\Lambda \mathfrak{g}_{1} \otimes N$. By 2.5.1] $F(N, I)$ is a free $A$-module whose rank is equal to the dimension of $\Lambda \mathfrak{g}_{1}$. Since $\widetilde{N}$ is a direct summand of $I$, the $A$-module $F(N, \widetilde{N})$ is a direct summand of $F(N, I)$. Hence $F(N, \tilde{N})$ is a finitely generated projective right $A$-module. One has $\operatorname{End}_{A}(F(N, \tilde{N}))=p \operatorname{End}_{A}(F(N, I)) p$ where $p \in \operatorname{End}_{A}(F(N, I))$ is the idempotent with the image $F(N, \tilde{N})$ corresponding to a decomposition $F(N, I)=F(N, \tilde{N}) \oplus$ $F(N, G)$. By 2.5.1 the left action of $F(I, I)$ on $F(N, I)$ induces an isomorphism $F(I, I) \stackrel{\sim}{\longrightarrow} \operatorname{End}_{A}(F(N, I))$. Thus the left action of $F(\tilde{N}, \tilde{N})$ on $F(N, \tilde{N})$ induces an isomorphism $F(\tilde{N}, \widetilde{N}) \stackrel{\sim}{\longrightarrow} \operatorname{End}_{A}(F(N, \tilde{N}))$. The assertion follows.

3.4.4. Retain the notation of 3.3 . Consider a canonical map $\operatorname{Ind}_{\mathfrak{g}_{0}}^{\mathfrak{g}} M \rightarrow \widetilde{M}$. By property (b) of ([6), this map is surjective. Recall $\widetilde{M}$ is projective in $\widetilde{\mathcal{O}}$. Consequently, $\widetilde{M}$ is a $\mathfrak{g}$-direct summand of $\operatorname{Ind}_{\mathfrak{g}_{0}}^{\mathfrak{g}} M$ and so the pair $(\widetilde{M}, M)$ satisfies the assumptions of Lemma 3.4.3. From 2.6 it follows that $F(\widetilde{M}, \widetilde{M}) \cong \tilde{\mathcal{U}} /(\tilde{\mathcal{U}} \widetilde{\chi})$ and $F(M, M) \cong \mathcal{U} /(\mathcal{U} \chi)$ as algebras. Taking into account Lemma 3.4.3 one concludes that the functor $\Phi^{\prime}: \mathcal{C}_{1} \rightarrow \widetilde{\mathcal{C}}_{1}$ defined by

$$
\Phi^{\prime}(N):=F(M, \widetilde{M}) \otimes_{\mathcal{U} /(\mathcal{U} \chi)} N=F(M, \widetilde{M}) \otimes_{\mathcal{U}} N
$$

provides an equivalence of the categories.

3.4.5. One has

$$
\Psi_{1} \circ \Phi^{\prime}(N)=F\left(M, \widetilde{M}_{\chi}\right) \otimes_{\mathcal{U}} N=F(M, M) \otimes_{\mathcal{U}} N=N
$$

since $F(M, M) \cong \mathcal{U} /(\mathcal{U} \chi)$. Hence $\Psi_{1}$ is quasi-inverse to $\Phi^{\prime}$ and it provides an equivalence of the categories $\widetilde{\mathcal{C}}_{1} \rightarrow \mathcal{C}_{1}$.

Let us verify that the functors $\Phi_{1}$ and $\Phi^{\prime}$ are isomorphic. Formula (5) implies that

$$
\Phi_{1}(N)=F\left(M,\left(\operatorname{Ind}_{\mathfrak{g}_{0}}^{\mathfrak{g}} M\right)_{\tilde{\chi}}\right) \otimes_{\mathcal{U}} N=F\left(M, \Phi_{1}(M)\right) \otimes_{\mathcal{U}} N
$$


for any $N \in \mathcal{C}_{1}$. Thus to show that $\Phi_{1}(N) \cong \Phi^{\prime}(N)$ for all $N \in \mathcal{C}_{1}$ it is enough to verify only that

$$
\left(\operatorname{Ind}_{\mathfrak{g}_{0}}^{\mathfrak{g}} M\right)_{\tilde{\chi}} \cong \widetilde{M}
$$

As we have shown above, $\widetilde{M}$ is a $\mathfrak{g}$-direct summand of $\operatorname{Ind}_{\mathfrak{g}_{0}}^{\mathfrak{g}} M$. Thus it is enough to check that

$$
\operatorname{Hom}_{\mathfrak{g}}\left(\tilde{N}, \operatorname{Ind}_{\mathfrak{g}_{0}}^{\mathfrak{g}} M\right)=0
$$

for all simple $\widetilde{N} \in \widetilde{\mathcal{C}}_{1}$ such that $\widetilde{N} \not$ Soc $\widetilde{M}$ and that

$$
\operatorname{dim} \operatorname{Hom}_{\mathfrak{g}}\left(\operatorname{Soc} \widetilde{M}, \operatorname{Ind}_{\mathfrak{g}_{0}}^{\mathfrak{g}} M\right)=1 .
$$

Recall that $\operatorname{Ind}_{\mathfrak{g}_{0}}^{\mathfrak{g}} M \cong \operatorname{Coind}_{\mathfrak{g}_{0}}^{\mathfrak{g}} M$ (see 2.8) and so

$$
\operatorname{Hom}_{\mathfrak{g}}\left(\tilde{N}, \operatorname{Ind}_{\mathfrak{g}_{0}}^{\mathfrak{g}} M\right)=\operatorname{Hom}_{\mathfrak{g}_{0}}(\tilde{N}, M)=\operatorname{Hom}_{\mathfrak{g}_{0}}\left(\widetilde{N}_{\chi}, M\right) .
$$

If $\tilde{N} \in \widetilde{\mathcal{C}}_{1}$ is simple, $\widetilde{N}_{\chi}=\Psi_{1}(\widetilde{N}) \in \mathcal{C}_{1}$ is also simple. Taking into account that the $\mathfrak{g}_{0}$-socle of $M$ is a simple Verma $\mathfrak{g}_{0}$-module, one concludes that $\operatorname{Hom}_{\mathfrak{g}_{0}}\left(\widetilde{N}_{\chi}, M\right)=0$ if $\widetilde{N}_{\chi} ¥ \operatorname{Soc} M$ and $\operatorname{dim} \operatorname{Hom}_{\mathfrak{g}_{0}}\left(\widetilde{N}_{\chi}, M\right)=1$ otherwise. If $\Psi_{1}(\widetilde{N})=\widetilde{N}_{\chi} \cong$ Soc $M$, then $\widetilde{N} \cong \operatorname{Soc} \widetilde{M}$ since $\Psi_{1}: \widetilde{\mathcal{C}}_{1} \rightarrow \mathcal{C}_{1}$ provides an equivalence of the categories and $\Psi_{1}(\widetilde{M})=M$. This proves (8) .

Hence the functors $\Phi_{1}$ and $\Phi^{\prime}$ are isomorphic. This completes the proof of Theorem 3.3.1.

\subsection{Remarks.}

3.5.1. Recall that any $\widetilde{N} \in \widetilde{\mathcal{C}}_{\infty}$ is generated by $\widetilde{N}_{\chi}$ and so is a homomorphic image of $\operatorname{Ind}_{\mathfrak{g}_{0}}^{\mathfrak{g}} \widetilde{N}_{\chi}$. Consequently, the restrictions of the functors $\Psi, \Phi$ provide an equivalence between $\widetilde{\mathcal{C}}_{\infty} \cap \widetilde{\mathcal{O}}$ and $\mathcal{C}_{\infty} \cap \mathcal{O}$. The same is true for the categories of weight modules. Also, one can easily see that the Verma modules of $\widetilde{\mathcal{C}}_{\infty} \cap \widetilde{\mathcal{O}}$ correspond to the Verma modules of $\mathcal{C}_{\infty} \cap \mathcal{O}$.

3.5.2. Formula (8), which says that $\Phi(M) \cong \widetilde{M}$, is crucial for the whole proof. If one had an independent proof of this formula, one could deduce from it a straightforward proof of Theorem 3.3.1.

3.6. Graded case. Denote by gr $\widetilde{\mathcal{C}}_{\infty}$ (resp., gr $\widetilde{\mathcal{C}}_{r}$ ) the full category of $\mathbb{Z}_{2}$-graded $\widetilde{\mathcal{U}}$-modules $\widetilde{N}$ satisfying $\widetilde{N}_{\tilde{\chi}}=\widetilde{N}$ (resp., $\widetilde{\chi}^{r} \widetilde{N}=0$ ). Consider $\mathcal{U}$ as a purely even superalgebra and define similarly $\operatorname{gr} \mathcal{C}_{\infty}, \operatorname{gr} \mathcal{C}_{r}$. Denote by $\#$ the forgetful functors $\operatorname{gr} \widetilde{\mathcal{C}}_{\infty} \rightarrow \widetilde{\mathcal{C}}_{\infty}$ and $\operatorname{gr} \mathcal{C}_{\infty} \rightarrow \mathcal{C}_{\infty}$

3.6.1. Evidently any $N^{\prime} \in \mathcal{C}_{\infty}$ is isomorphic to $N^{\#}$ for some $N \in \operatorname{gr} \mathcal{C}_{\infty}$ - for instance, one can consider $N^{\prime}$ as a purely even (odd) module. It turns out that a similar assertion holds for $\widetilde{\mathcal{C}}_{\infty}$. Indeed, since $\widetilde{\chi}$ is strongly typical, it contains an element $\left(T^{2}-c^{2}\right)$ for some non-zero $c \in \mathbb{C}$. Denote by $\widetilde{\mathcal{U}}_{0}$ the even part of $\widetilde{\mathcal{U}}$; recall that $T$ lies in the centre of $\widetilde{\mathcal{U}}_{0}$. Any $\widetilde{N} \in \widetilde{\mathcal{C}}_{\infty}$ is a direct sum of the $\widetilde{\mathcal{U}}_{0}$-modules $N_{+}$, $N_{-}$where

$$
\begin{aligned}
& N_{+}:=\left\{v \in \widetilde{M} \mid(T-c)^{r} v=0, \forall r>>0\right\}, \\
& N_{-}:=\left\{v \in \widetilde{M} \mid(T+c)^{r} v=0, \forall r>>0\right\} .
\end{aligned}
$$




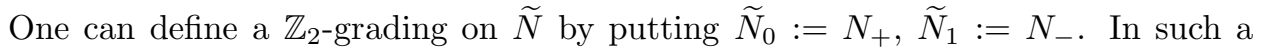
way, one obtains a functor $\widetilde{\mathcal{C}}_{\infty} \rightarrow \operatorname{gr} \widetilde{\mathcal{C}}_{\infty}$ which is left quasi-inverse to the functor \#. This implies, in particular, that for any irreducible graded $\widetilde{\mathcal{U}} /(\widetilde{\mathcal{U}} \widetilde{\chi})$-module $\widetilde{N}$, its image $\widetilde{N}^{\#}$ remains an irreducible (non-graded) module.

\subsubsection{Theorem. The functors}

$$
\begin{aligned}
& \Psi^{\mathrm{gr}}: \operatorname{gr} \widetilde{\mathcal{C}}_{\infty} \rightarrow \operatorname{gr} \mathcal{C}_{\infty}, \quad \tilde{N} \mapsto \widetilde{N}_{\chi}, \\
& \Phi^{\mathrm{gr}}: \operatorname{gr} \mathcal{C}_{\infty} \rightarrow \operatorname{gr} \widetilde{\mathcal{C}}_{\infty}, \quad N \mapsto\left(\operatorname{Ind}_{\mathfrak{g}_{0}}^{\mathfrak{g}} N\right)_{\tilde{\chi}}
\end{aligned}
$$

are mutually quasi-inverse. Their restriction provides an equivalence of the categories $\operatorname{gr} \widetilde{\mathcal{C}}_{r}$ and $\operatorname{gr} \mathcal{C}_{r}$ for any $r \in \mathbb{N}^{+}$.

Proof. Repeating the arguments of Lemma 3.2.1, one shows that the functor $\Psi^{\mathrm{gr}}$ is left and right adjoint to the functor $\Phi^{\mathrm{gr}}$.

The functors $\Psi, \Phi$ are quasi-inverse and so the canonical homomorphism $\alpha_{N}$ : $N \rightarrow \Psi \circ \Phi(N)$ is an isomorphism for any $N \in \mathcal{C}_{\infty}$. For any $N \in \operatorname{gr} \mathcal{C}_{\infty}$ denote by $\alpha_{N}$ the canonical homomorphism $N \rightarrow \Psi^{\mathrm{gr}} \circ \Phi^{\mathrm{gr}}(N)$. One has $\# \circ \Psi^{\mathrm{gr}}=\Psi \circ \#$ and $\# \circ \Phi^{\mathrm{gr}}=\Phi \circ \#$. Therefore \# $\left(\alpha_{N}\right)=\alpha_{N \#}$. Since $\alpha_{N \#}$ is an isomorphism, $\alpha_{N}$ is also an isomorphism. Similarly, the canonical homomorphism $\beta_{\widetilde{N}}: \Phi^{\mathrm{gr}} \circ \Psi^{\mathrm{gr}}(\widetilde{N}) \rightarrow \widetilde{N}$ is an isomorphism for any $\widetilde{N} \in \operatorname{gr} \widetilde{\mathcal{C}}_{\infty}$. Hence gr $\Psi$ and gr $\Phi$ are quasi-inverse.

The equality $\Psi^{\mathrm{gr}}\left(\operatorname{gr} \widetilde{\mathcal{C}_{r}}\right)=\operatorname{gr} \mathcal{C}_{r}$ follows from the equality $\Psi\left(\operatorname{gr} \widetilde{\mathcal{C}}_{r}\right)=\mathcal{C}_{r}$. This implies the second claim.

\section{An example}

Say that a $\tilde{\mathcal{U}}$-central character is weakly atypical if it is not strongly typical. In this section we consider a "generic" weakly atypical central character for $\mathfrak{g}:=$ $\mathfrak{o} \mathfrak{s p}(1,2 l)$. We show that, for such a character $\mathfrak{m} \in \operatorname{Max} \mathcal{Z}(\mathfrak{g})$, the category $\operatorname{gr} \widetilde{\mathcal{C}}_{\infty}$ of graded $\mathfrak{g}$-modules $\widetilde{N}$ satisfying $\widetilde{N}_{\mathfrak{m}}=\widetilde{N}$ is equivalent to the category $\mathcal{C}_{\infty}$ of $\mathfrak{g}_{0}$-modules $N$ satisfying $N_{\chi}=N$ for an appropriate $\chi$. We see that in this case the category gr $\widetilde{\mathcal{C}}_{\infty}$ is "twice smaller" than one could expect in the strongly typical case.

Throughout this section all $\mathfrak{g}$-modules are assumed to be graded.

4.1. The superalgebra $\mathfrak{o s p}(1,2 l)$ does not have isotropic roots: $(\beta, \beta) \neq 0$ for any $\beta \in \Delta_{1}$. As a result, osp $(1,2 l)$ has many features of the simple Lie algebras. For instance, $\widetilde{\mathcal{U}}$ is a domain, its centre $\mathcal{Z}(\mathfrak{g})$ is a polynomial algebra and all finitedimensional representations are completely reducible. In [M1, Musson proved the existence of a harmonic space $H$ in $\widetilde{\mathcal{U}}$; this is an ad $\mathfrak{g}$-submodule of $\widetilde{\mathcal{U}}$ such that the multiplication map provides the isomorphism $H \otimes \mathcal{Z}(\mathfrak{g}) \stackrel{\sim}{\longrightarrow} \widetilde{\mathcal{U}}$.

If $\widetilde{M}$ is strongly typical, then its annihilator is a centrally generated ideal.

Suppose that $\widetilde{M}$ is not strongly typical; this means that $\mathfrak{m}:=\operatorname{Ann}_{\mathcal{Z}(\mathfrak{g})} \widetilde{M}$ contains $T^{2}$. Then, by [GL1], 6.2,

$$
\text { Ann } \widetilde{M}=\widetilde{\mathcal{U}}(T \mathcal{Z}(\mathfrak{g})+\mathfrak{m})
$$

and $\tilde{\chi}:=(T \mathcal{Z}(\mathfrak{g})+\mathfrak{m})$ is a maximal ideal of the "ghost centre" $\widetilde{\mathcal{Z}}(\mathfrak{g})=\mathcal{Z}(\mathfrak{g})+T \mathcal{Z}(\mathfrak{g})$. The ideal $\widetilde{\mathcal{U}} \widetilde{\chi}$ is primitive since a Verma module $\widetilde{M}(\mu)$ is simple if $\mu \in W(\mathfrak{m})$ is minimal. 
Moreover, by [GL1], 5.3, for any simple $\mathfrak{g}_{0}$-module $V$ one has

$$
\operatorname{dim} \operatorname{Hom}_{\mathfrak{g}_{0}}(V, \widetilde{\mathcal{U}} /(\operatorname{Ann} \widetilde{M}))=\frac{1}{2} \operatorname{dim} \operatorname{Hom}_{\mathfrak{g}_{0}}(V, H)
$$

where $H$ is the harmonic space mentioned above.

4.2. Retain the notation of 2.4.2. Recall that $\mathfrak{m} \in \operatorname{Max} \mathcal{Z}(\mathfrak{g})$ is strongly typical iff the elements $\lambda \in W(\mathfrak{m})$ do not belong to the hyperplanes

$$
S_{\beta}:=\left\{\mu \in \mathfrak{h}^{*} \mid(\mu+\rho, \beta)=0\right\}
$$

for $\beta \in \Delta_{1}^{+}$. In this section we consider $\mathfrak{m} \in \operatorname{Max} \mathcal{Z}(\mathfrak{g})$ such that any $\lambda \in W(\mathfrak{m})$ belongs to exactly one hyperplane $S_{\beta}$, that is,

$$
\exists ! \beta \in \Delta_{1}^{+}: \quad(\lambda+\rho, \beta)=0 .
$$

In particular, $\mathfrak{m}$ is not strongly typical and so $T^{2} \in \mathfrak{m}$. Set

$$
\tilde{\chi}:=T \mathcal{Z}(\mathfrak{g})+\mathfrak{m} .
$$

For any $\mathfrak{g}$-module $\widetilde{N}$ the vector space

$$
\widetilde{N}_{\tilde{\chi}}:=\left\{v \in \tilde{N} \mid \widetilde{\chi}^{r} v=0, \quad r>>0\right\}
$$

is a $\mathfrak{g}$-submodule of $\widetilde{N}$. Let gr $\widetilde{\mathcal{C}}_{r}$ (resp., gr $\widetilde{\mathcal{C}}_{\infty}$ ) be the category of graded $\mathfrak{g}$-modules $\widetilde{N}$ satisfying $\widetilde{\chi}^{r} \widetilde{N}=0$ (resp., $\widetilde{N}_{\tilde{\chi}}=\widetilde{N}$ ). Observe that $\operatorname{gr} \widetilde{\mathcal{C}}_{1} \subseteq \operatorname{gr} \widetilde{\mathcal{C}}_{1}^{\prime} \subseteq$ gr $\widetilde{\mathcal{C}}_{2}$ where $\operatorname{gr} \widetilde{\mathcal{C}}_{1}^{\prime}$ is the category of graded $\mathfrak{g}$-modules $\widetilde{N}$ satisfying $\mathfrak{m} \tilde{N}=0$.

Fix a projective Verma module $\widetilde{M}$ annihilated by $\widetilde{\chi}$. Fix $\chi \in \operatorname{Max} \mathcal{Z}\left(\mathfrak{g}_{0}\right)$ such that

$$
\begin{aligned}
& \text { (a) } M:=\widetilde{M}_{\chi ; 0} \text { is a Verma } \mathfrak{g}_{0} \text {-module, } \\
& \text { (b) } \widetilde{M}=\widetilde{\mathcal{U}} M
\end{aligned}
$$

(the existence of $\chi$ satisfying (11) will be shown in 4.5). Let $\mathcal{C}_{\infty}$ (resp., $\mathcal{C}_{r}$ ) be the category of $\mathfrak{g}_{0}$-modules $N$ satisfying $N_{\chi}=N$ (resp., $\chi^{r} N=0$ ).

4.3. Theorem. The functors

$$
\begin{array}{ll}
\Psi: \operatorname{gr} \widetilde{\mathcal{C}_{\infty}} \rightarrow \mathcal{C}_{\infty}, & \widetilde{N} \mapsto\left(\widetilde{N}_{0}\right)_{\chi}, \\
\Phi: \mathcal{C}_{\infty} \rightarrow \operatorname{gr} \widetilde{\mathcal{C}} \infty, & N \mapsto\left(\operatorname{Ind}_{\mathfrak{g}_{0}}^{\mathfrak{g}} N\right)_{\tilde{\chi}}
\end{array}
$$

where the grading on $\operatorname{Ind}_{\mathfrak{g}_{0}}^{\mathfrak{g}} N=\widetilde{\mathcal{U}} \otimes \mathcal{U} N$ is determined by assuming $N$ to be even, are quasi-inverse. Moreover their restrictions to gr $\widetilde{\mathcal{C}}_{r}$ and $\mathcal{C}_{r}$ respectively provide an equivalence of these categories.

Proof. The first steps of the proof are the same as those in the proof of Theorem 3.3.1. Considering $\operatorname{Ind}_{\mathfrak{g}_{0}}^{\mathfrak{g}} N$ as the graded module with respect to the grading defined above one obtains

$$
\operatorname{Hom}_{\mathfrak{g}}\left(\operatorname{Ind}_{\mathfrak{g}_{0}}^{\mathfrak{g}} N, \widetilde{N}\right)=\operatorname{Hom}_{\mathfrak{g}_{0}}\left(N, \widetilde{N}_{0}\right)
$$

for any graded $\mathfrak{g}$-module $\widetilde{N}$. Repeating 3.2.1, one concludes that the functors $\Psi, \Phi$ are adjoint. Repeating 3.4.1, one reduces the required assertion to "the case $r=1$ ", that is, to the statement that the restrictions $\Psi_{1}, \Phi_{1}$ of the functors $\Psi, \Phi$ to the categories gr $\widetilde{\mathcal{C}}_{1}, \mathcal{C}_{1}$ provide an equivalence of the categories.

Evidently, $M$ is a $\mathfrak{g}_{0}$-direct summand of $\widetilde{M}$. The embedding $M \rightarrow \widetilde{M}$ gives rise to a non-zero $\mathfrak{g}$-map $\operatorname{Ind}_{\mathfrak{g}_{0}}^{\mathfrak{g}} M \rightarrow \widetilde{M}$. Using $\widetilde{M}=\widetilde{\mathcal{U}} M$ and the projectivity 
of $\widetilde{M}$, one concludes that $\widetilde{M}$ is a $\mathfrak{g}$-direct summand of $\operatorname{Ind}_{\mathfrak{g}_{0}}^{\mathfrak{g}} M$. Denote by $B$ the algebra $F(\widetilde{M}, \widetilde{M})$ and by $B$-Mod the category of $B$-modules. Taking into account Lemma 3.4.3. one concludes that the functor $\Phi^{\prime \prime}: \mathcal{C}_{1} \rightarrow B$-Mod defined by

$$
\Phi^{\prime \prime}(N):=F(M, \widetilde{M}) \otimes_{\mathcal{U}} N
$$

provides an equivalence of the categories.

The category gr $\widetilde{\mathcal{C}_{1}}$ is the category of graded modules over the superalgebra $A:=$ $\widetilde{\mathcal{U}} /(\widetilde{\mathcal{U}} \widetilde{\chi})$. In Proposition 4.7 below we prove that $B=A \oplus A \theta$ where $\theta \in F(\widetilde{M}, \widetilde{M})$ is given by

$$
\theta(v)=(-1)^{d(v)} v
$$

In particular, $\theta^{2}=1$ and $\theta a=(-1)^{d(a)} a \theta$ for any $a \in A$. Define the functor Gr $: B$ $\operatorname{Mod} \rightarrow \operatorname{gr} \widetilde{\mathcal{C}_{1}}$ as follows: for any $X \in B$-Mod the $A$-module structure on $\operatorname{Gr}(X)$ is given by "the restriction of scalars", and the grading is given by

$$
\operatorname{Gr}(X)_{0}:=\{v \in X \mid \theta v=v\}, \operatorname{Gr}(X)_{1}:=\{v \in X \mid \theta v=-v\} .
$$

It is easy to see that Gr provides an equivalence of the categories $B$-Mod and $\operatorname{gr} \widetilde{\mathcal{C}_{1}}$. Hence $\Phi^{\prime}:=\operatorname{Gr} \circ \Phi^{\prime \prime}$ provides an equivalence of the categories $\mathcal{C}_{1}$ and gr $\widetilde{\mathcal{C}}_{1}$.

The functor $\Phi^{\prime}: \mathcal{C}_{1} \rightarrow \operatorname{gr} \widetilde{\mathcal{C}}_{1}$ is given by the formula $N \mapsto F(M, \widetilde{M}) \otimes \mathcal{U} N$ where the grading on $F(M, \widetilde{M})$ is defined by $F(M, \widetilde{M})_{i}:=F\left(M, \widetilde{M}_{i}\right)(i=0,1)$.

The last step of the proof repeats 3.4.5 Indeed,

$$
\Psi_{1} \circ \Phi^{\prime}(N)=F\left(M, \widetilde{M}_{\chi ; 0}\right) \otimes_{\mathcal{U}} N=F(M, M) \otimes_{\mathcal{U}} N=N
$$

since $F(M, M) \cong \mathcal{U} /(\mathcal{U} \chi)$. Hence $\Psi_{1}$ is quasi-inverse to $\Phi^{\prime}$ and it provides an equivalence of the categories $\widetilde{\mathcal{C}}_{1} \rightarrow \mathcal{C}_{1}$. Now, in order to verify that the functors $\Phi_{1}$ and $\Phi^{\prime}$ are isomorphic, one can simply repeat 3.4 .5 for the graded modules.

4.4. The restrictions of the functors $\Psi, \Phi$ provide an equivalence between $\widetilde{\mathcal{C}}_{\infty} \cap \widetilde{\mathcal{O}}$ and $\mathcal{C}_{\infty} \cap \mathcal{O}$ and between the corresponding categories of weight modules. The Verma modules of $\widetilde{\mathcal{C}}_{\infty} \cap \widetilde{\mathcal{O}}$ correspond to the Verma modules of $\mathcal{C}_{\infty} \cap \mathcal{O}$.

4.4.1. The category gr $\widetilde{\mathcal{C}}_{\infty}$ has a canonical involution $\Pi$ given by

$$
(\Pi \widetilde{N})_{0}=\widetilde{N}_{1}, \quad(\Pi \widetilde{N})_{1}=\widetilde{N}_{0} .
$$

This leads to an interesting involution $\Pi^{\prime}=\Psi \circ \Pi \circ \Phi$ on the category $\mathcal{C}_{\infty}$. One has

$$
\begin{aligned}
N & =\left(\left(\operatorname{Ind}_{\mathfrak{g}_{0}}^{\mathfrak{g}} N\right)_{\tilde{\chi}}\right)_{\chi ; 0}, \\
\Pi^{\prime}(N) & =\left(\left(\operatorname{Ind}_{\mathfrak{g}_{0}}^{\mathfrak{g}} N\right)_{\tilde{\chi}}\right)_{\chi ; 1} .
\end{aligned}
$$

Theorem 4.3 implies that $M^{\prime}:=\widetilde{M}_{\chi ; 1}=\Psi(\Pi(\widetilde{M}))$ is a Verma $\mathfrak{g}_{0}$-module. For $N \in \mathcal{C}_{1}$ one has $\Phi(N) \cong \Phi^{\prime}(N)$ and so

$$
\Pi^{\prime}(N)=F\left(M, M^{\prime}\right) \otimes_{\mathcal{U}} N .
$$

4.5. It remains to prove Proposition 4.7 and to find $\chi \in \operatorname{Max} \mathcal{Z}\left(\mathfrak{g}_{0}\right)$ satisfying (11). To achieve these goals we proceed in a manner similar to G2], Section 8 .

Call $\chi \in \operatorname{Max} \mathcal{Z}\left(\mathfrak{g}_{0}\right)$ a mate for $\tilde{\chi}$ if for a Verma module $\widetilde{M}$ annihilated by $\widetilde{\chi}$ the $\mathfrak{g}_{0}$-modules $\widetilde{M}_{\chi ; 0}, \widetilde{M}_{\chi ; 1}$ are Verma modules. Call $\chi \in \operatorname{Max} \mathcal{Z}\left(\mathfrak{g}_{0}\right)$ a perfect mate for $\tilde{\chi}$ if it is a mate and for a simple highest weight module $\widetilde{V}(\lambda)$ annihilated by $\tilde{\chi}$ the $\mathfrak{g}_{0}$-module $\widetilde{V}(\lambda)_{\chi ; 0}$ is non-zero.

In this subsection we construct a perfect mate $\chi$ for $\tilde{\chi}$ satisfying (10). 
4.5.1. The root system of $\mathfrak{g}$ takes the form

$$
\Delta_{1}^{+}=\left\{\sigma_{i}\right\}_{1 \leq i \leq l}, \quad \Delta_{0}^{+}=\left\{\sigma_{i} \pm \sigma_{j} ; 2 \sigma_{i}\right\}_{1 \leq i<j \leq l}
$$

and $\left(\sigma_{i}, \sigma_{j}\right)=\delta_{i, j}$. The Weyl group $W$ acts on $\left\{\sigma_{i}\right\}_{1}^{l}$ by the signed permutations.

Set

$$
\begin{aligned}
\Gamma: & =\left\{\sum_{1}^{l} r_{i} \sigma_{i} \mid r_{i} \in\{0,1\}\right\}, \\
\Gamma_{0}: & =\left\{\sum_{1}^{l} r_{i} \sigma_{i} \mid r_{i} \in\{0,1\}, \sum_{1}^{l} r_{i} \text { is even }\right\}, \\
\Gamma_{1}: & =\left\{\sum_{1}^{l} r_{i} \sigma_{i} \mid r_{i} \in\{0,1\}, \sum_{1}^{l} r_{i} \text { is odd }\right\} .
\end{aligned}
$$

Define the action of the Weyl group $W$ on $\Gamma$ by setting

$$
w_{*} \gamma=w\left(\gamma-\rho_{1}\right)+\rho_{1} .
$$

Take an arbitrary $\lambda \in \mathfrak{h}^{*}$ and fix a $\mathbb{Z}_{2}$-grading on a Verma module $\widetilde{M}(\lambda)$ in such a way that a highest weight vector becomes even. As a $\mathfrak{g}_{0}$-module, $\widetilde{M}=\widetilde{M}_{0} \oplus \widetilde{M}_{1}$; the module $\widetilde{M}(\lambda)_{i}$ has a filtration such that the set of factors coincides with the set $\left\{M(\lambda-\gamma): \gamma \in \Gamma_{i}\right\}$ - see M1], 3.2.

It is easy to check that for any $w \in W, \gamma \in \Gamma$

$$
w \cdot \lambda-w_{*} \gamma+\rho_{0}=w\left(\lambda-\gamma+\rho_{0}\right) .
$$

Therefore the $\mathfrak{g}_{0}$-central characters of $M\left(w \cdot \lambda-w_{*} \gamma\right)$ and $M(\lambda-\gamma)$ coincide. Thus the multiset of $\mathfrak{g}_{0}$-central characters of $\{M(w \cdot \lambda-\gamma): \gamma \in \Gamma\}$ does not depend on the choice of $w \in W$. Recall that the set

$$
W(\widetilde{\chi}):=\left\{\mu \in \mathfrak{h}^{*} \mid \widetilde{\chi} \widetilde{M}(\mu)=0\right\}
$$

forms a single $W$.-orbit.

4.5.2. Say that $\mu, \mu^{\prime} \in \sum_{i} \mathbb{Z} \sigma_{i}$ have the same parity if $\left(\mu-\mu^{\prime}\right) \in \mathbb{Z} \Delta_{0}^{+}$. Suppose that $\gamma, \gamma^{\prime} \in \Gamma$ both lie either in $\Gamma_{0}$ or in $\Gamma_{1}$. This is equivalent to the condition that the elements $\gamma, \gamma^{\prime}$ have the same parity. For any $w \in W$ one has $w_{*} \gamma-$ $w_{*} \gamma^{\prime}=w\left(\gamma-\gamma^{\prime}\right)$ and so the elements $w_{*} \gamma, w_{*} \gamma^{\prime}$ also have the same parity. Hence both $w_{*} \gamma, w_{*} \gamma^{\prime}$ lie either in $\Gamma_{0}$ or in $\Gamma_{1}$. This has the following consequence: the multiset of $\mathfrak{g}_{0}$-central characters of $\left\{M(w \cdot \lambda-\gamma): \gamma \in \Gamma_{0}\right\}$ coincides either with the multiset of $\mathfrak{g}_{0}$-central characters of $\left\{M(\lambda-\gamma): \gamma \in \Gamma_{0}\right\}$ or with the multiset of $\mathfrak{g}_{0}$-central characters of $\left\{M(\lambda-\gamma): \gamma \in \Gamma_{1}\right\}$. In particular, if $\chi \in \operatorname{Max} \mathcal{Z}\left(\mathfrak{g}_{0}\right)$ is such that for some $\lambda \in W(\widetilde{\chi})$ one has $\widetilde{M}(\lambda)_{\chi}=M\left(\lambda-\gamma_{0}\right) \oplus M\left(\lambda-\gamma_{1}\right)$ for certain $\gamma_{i} \in \Gamma_{i}(i=0,1)$, then for any $\lambda^{\prime} \in W(\widetilde{\chi})$ the $\mathfrak{g}_{0}$-modules $\widetilde{M}\left(\lambda^{\prime}\right)_{\chi ; i}$ are Verma modules.

4.5.3. Define a lexicographic order on $\mathbb{C}$ by setting $c_{1}>c_{2}$ if $\operatorname{Re} c_{1}>\operatorname{Re} c_{2}$ or $\operatorname{Re} c_{1}=\operatorname{Re} c_{2}$ and $\operatorname{Im} c_{1}>\operatorname{Im} c_{2}$.

The condition (10) implies the existence of $\lambda \in W(\widetilde{\chi})$ such that

$$
\lambda+\rho=\sum_{1}^{l} k_{i} \sigma_{i}, \quad k_{1}, \ldots, k_{l-1}>k_{l}=0 .
$$


Set $\widetilde{M}:=\widetilde{M}(\lambda)$ and

$$
\chi=\operatorname{Ann}_{\mathcal{Z}\left(\mathfrak{g}_{0}\right)} M(\lambda) .
$$

One has $\rho_{1}=\frac{1}{2} \sum_{1}^{l} \sigma_{i}$ and so

$$
\lambda+\rho_{0}=\sum_{1}^{l}\left(k_{i}+\frac{1}{2}\right) \sigma_{i}
$$

One easily sees that

$$
\lambda+\rho_{0}-\gamma \in W\left(\lambda+\rho_{0}\right)
$$

only for $\gamma=\sigma_{l}, 0$. Therefore $\chi M(\lambda-\gamma)=0$ iff $\gamma=\sigma_{l}, 0$. This implies $\widetilde{M}_{\chi}=$ $M(\lambda) \oplus M\left(\lambda-\sigma_{l}\right)$. By 4.5.2, $\chi$ is a mate for $\tilde{\chi}$.

4.5.4. Let us show that $\chi$ is a perfect mate. Suppose this is not true. Then $\widetilde{V}(w \cdot \lambda)_{\chi ; i}=0$ for some $w \in W$ and $i \in\{0,1\}$. Equality (12) implies that for any $y \in W$

$$
\widetilde{M}(y \cdot \lambda)_{\chi}=M\left(y \cdot \lambda-y_{*} 0\right) \oplus M\left(\lambda-y_{*} \sigma_{l}\right)
$$

and so $\tilde{V}(y \cdot \lambda)_{\chi}$ is a homomorphic image of $M\left(y \cdot \lambda-y_{*} 0\right) \oplus M\left(\lambda-y_{*} \sigma_{l}\right)$. The module $\widetilde{V}(w \cdot \lambda)$ is a homomorphic image of the module $\widetilde{M}(w \cdot \lambda)$; denote the kernel of this homomorphism by $\widetilde{N}$. The module $\widetilde{N}$ has finite length and the factors of its Jordan-Gölder series have form $\widetilde{V}(\mu)$ for some $\mu \in W \cdot \lambda$ satisfying $\mu<w . \lambda$. Since $0=\widetilde{V}(w \cdot \lambda)_{\chi ; i}=(\widetilde{M}(w \cdot \lambda) / \widetilde{N})_{\chi ; i}$, one concludes that the $\mathfrak{g}_{0}$-module $\widetilde{N}_{\chi ; i}=$ $\widetilde{M}(w \cdot \lambda)_{\chi ; i}$ has a finite filtration whose factors are quotients of either $M\left(y \cdot \lambda-y_{*} 0\right)$ or $M\left(y . \lambda-y_{*} \sigma_{l}\right)$ for some $y \in W$ satisfying $y \cdot \lambda<w . \lambda$. Hence the highest weight of $\widetilde{M}(w \cdot \lambda)_{\chi ; i}$ belongs to the set

$$
X:=\left\{y \cdot \lambda-y_{*} 0, y \cdot \lambda-y_{*} \sigma_{l}\right\}_{y \in W} \text { s.t. } y \cdot \lambda<w . \lambda .
$$

The module $\widetilde{M}(w \cdot \lambda)_{\chi ;_{i}}$ is isomorphic either to $M\left(w \cdot \lambda-w_{*} 0\right)$ or to $M\left(w \cdot \lambda-w_{*} \sigma_{l}\right)$. Therefore either $w \cdot \lambda-w_{*} 0$ or $w \cdot \lambda-w_{*} \sigma_{l}$ belongs to the set $X$.

If $w \cdot \lambda-w_{*} 0=y \cdot \lambda-y_{*} 0$, then, by [12), $w\left(\lambda+\rho_{0}\right)=y\left(\lambda+\rho_{0}\right)$, that is, $w^{-1} y \in \operatorname{Stab}_{W}\left(\lambda+\rho_{0}\right)$. One has

$$
\operatorname{Stab}_{W}\left(\lambda+\rho_{0}\right)=\operatorname{Stab}_{W}\left(\sum_{1}^{l}\left(k_{j}+\frac{1}{2}\right) \sigma_{j}\right) \subseteq \operatorname{Stab}_{W}\left(\sum_{1}^{l} k_{j} \sigma_{j}\right)=\operatorname{Stab}_{W}(\lambda+\rho)
$$

since $k_{1}, \ldots, k_{l-1}>k_{l}=0$. Thus $w \cdot \lambda-w_{*} 0=y \cdot \lambda-y_{*} 0$ implies $w \cdot \lambda=y \cdot \lambda$. Hence $\left(w \cdot \lambda-w_{*} 0\right) \notin\left\{y \cdot \lambda-y_{*} 0\right\}_{y \in W, y \cdot \lambda<w . \lambda}$.

If $w \cdot \lambda-w_{*} 0=y \cdot \lambda-y_{*} \sigma_{l}$, then, by (12), $w\left(\lambda+\rho_{0}\right)=y\left(\lambda+\rho_{0}-\sigma_{l}\right)$ or, equivalently, $w\left(\lambda+\rho_{0}\right)=y s_{\beta_{l}}\left(\lambda+\rho_{0}\right)$ where $s_{\beta_{l}} \in W$ is the reflection with respect to the root $\beta_{l}$. Therefore $w^{-1} y s_{\beta_{l}} \in \operatorname{Stab}_{W}\left(\lambda+\rho_{0}\right)$. As we already saw $\operatorname{Stab}_{W}\left(\lambda+\rho_{0}\right) \subseteq$ $\operatorname{Stab}_{W}(\lambda+\rho)$ and so $w^{-1} y s_{\beta_{l}} \in \operatorname{Stab}_{W}(\lambda+\rho)$. Then $w^{-1} y \in \operatorname{Stab}_{W}(\lambda+\rho)$ since $s_{\beta_{l}} \in \operatorname{Stab}_{W}(\lambda+\rho)$. Hence $w \cdot \lambda-w_{*} 0=y \cdot \lambda-y_{*} \sigma_{l}$ forces $w^{-1} y \in \operatorname{Stab}_{W}(\lambda+\rho)$. We conclude that $\left(w \cdot \lambda-w_{*} 0\right) \notin X$.

Similarly, if $w \cdot \lambda-w_{*} \sigma_{l}=y \cdot \lambda-y_{*} 0$, then, by (12), $w\left(\lambda+\rho_{0}-\sigma_{l}\right)=y\left(\lambda+\rho_{0}\right)$. As we have shown above, this implies $w^{-1} y \in \operatorname{Stab}_{W}(\lambda+\rho)$. Hence $\left(w \cdot \lambda-w_{*} \sigma_{l}\right) \notin$ $\left\{y \cdot \lambda-y_{*} 0\right\}_{y \in W, y \cdot \lambda<w . \lambda}$. 
Finally, if $w \cdot \lambda-w_{*} \sigma_{l}=y \cdot \lambda-y_{*} \sigma_{l}$, then, by (12), $w^{-1} y \in \operatorname{Stab}_{W}\left(\lambda+\rho_{0}-\sigma_{l}\right)$. One can easily deduce from the equality

$$
\lambda+\rho_{0}-\sigma_{l}=\sum_{1}^{l-1}\left(k_{j}+\frac{1}{2}\right) \sigma_{j}-\frac{1}{2} \sigma_{l}
$$

that $\operatorname{Stab}_{W}\left(\lambda+\rho_{0}-\sigma_{l}\right) \subseteq \operatorname{Stab}_{W}(\lambda+\rho)$. Thus $\left(w \cdot \lambda-w_{*} \sigma_{l}\right) \notin X$ as required.

Hence $\left\{w \cdot \lambda-w_{*} 0 ; w \cdot \lambda-w_{*} \sigma_{l}\right\} \cap X=\emptyset$. This proves that $\widetilde{V}(w \cdot \lambda)_{\chi ; i} \neq 0$ for $i=0,1$.

4.5.5. Corollary. The ideal $\chi \in \operatorname{Max} \mathcal{Z}\left(\mathfrak{g}_{0}\right)$ described in 4.5.3 is a perfect mate for $\tilde{\chi}$.

4.6. Suppose that $\chi \in \operatorname{Max} \mathcal{Z}\left(\mathfrak{g}_{0}\right)$ is a perfect mate for $\tilde{\chi}$.

The ideal $\widetilde{\mathcal{U}} \widetilde{\chi}$ is equal to the annihilator of a Verma module $\widetilde{M}$ which has a finite support in $\mathcal{Z}\left(\mathfrak{g}_{0}\right)$. Therefore any $\mathfrak{g}$-module annihilated by $\widetilde{\mathcal{U}} \widetilde{\chi}$ has a finite support in $\mathcal{Z}\left(\mathfrak{g}_{0}\right)$. Arguing as in $\mathrm{G} 2,8.3 .2,8.3 .3$, one can deduce from the definition of perfect mate that for a graded $\mathfrak{g}$-module $\widetilde{N}$ annihilated by $\widetilde{\mathcal{U}} \widetilde{\chi}$ one has $\widetilde{N}=\widetilde{\mathcal{U}} \widetilde{N}_{\chi ; 0}$. In particular, $\widetilde{N}_{\chi ; 0} \neq 0$ if $\widetilde{N} \neq 0$.

For a graded $\mathfrak{g}$-bimodule $L$ set

$$
{ }_{\chi} L_{\chi ; 0}:=\left\{f \in L_{0} \mid \chi^{r} f=f \chi^{r}=0, \forall r>>0\right\} .
$$

Arguing as in [G2], 8.4, one concludes ${ }_{\chi} L_{\chi ; 0} \neq 0$ provided $L \neq 0$ and $\tilde{\chi} L=L \tilde{\chi}=0$.

4.7. Proposition. Let $\widetilde{M}$ be a Verma module annihilated by $\tilde{\chi}$ and let $A$ be the image of $\widetilde{\mathcal{U}}$ in $F(\widetilde{M}, \widetilde{M})$ under the natural map. Then

$$
F(\widetilde{M}, \widetilde{M})=A \oplus A \theta
$$

where $\theta \in F(\widetilde{M}, \widetilde{M})$ is given by

$$
\theta(v)=(-1)^{d(v)} v .
$$

Proof. Suppose that $\widetilde{M}$ is simple. By [G2], 11.1.5, for any simple $\mathfrak{g}$-module $\widetilde{V}$ one has $\operatorname{dim} \operatorname{Hom}_{\mathfrak{g}}(\widetilde{V}, F(\widetilde{M}, \widetilde{M}))=\operatorname{dim}_{\operatorname{Hom}}(\widetilde{V}, H)$ where $H$ is a harmonic space see 4.1 The complete reducibility of both $H$ and $F(\widetilde{M}, \widetilde{M})$ forces $H \cong F(\widetilde{M}, \widetilde{M})$. The element $\theta$ is ad $\mathfrak{g}_{0}$-invariant and so $A \cong A \theta$ as ad $\mathfrak{g}_{0}$-modules. In light of (9), as ad $\mathfrak{g}_{0}$-modules $H \cong A \oplus A$. Since the multiplicity of any finite-dimensional $\mathfrak{g}_{0^{-}}$ module in $H$ is finite, in order to prove the equality $F(\widetilde{M}, \widetilde{M})=A \oplus A \theta$ it is enough to show that $F(\widetilde{M}, \widetilde{M})=A+A \theta$. Since $\theta u=(-1)^{d(u)} u \theta$ for any $u \in \widetilde{\mathcal{U}},(A+A \theta)$ is a graded $\widetilde{\mathcal{U}}$-subbimodule of $F(\widetilde{M}, \widetilde{M})$. Let $\chi \in \mathcal{Z}\left(\mathfrak{g}_{0}\right)$ be a perfect mate for $\widetilde{\chi}$. Then both $M:=\widetilde{M}_{\chi ; 0}$ and $M^{\prime}:=\widetilde{M}_{\chi ; 1}$ are Verma $\mathfrak{g}_{0}$-modules. The restriction of endomorphisms of $\widetilde{M}$ to $M \oplus M^{\prime}$ induces an isomorphism

$$
{ }_{\chi} F(\widetilde{M}, \widetilde{M})_{\chi ; 0} \stackrel{\sim}{\longrightarrow} F(M, M) \oplus F\left(M^{\prime}, M^{\prime}\right) .
$$

Recall that the natural maps $\mathcal{U} /(\mathcal{U} \chi) \rightarrow F(M, M)$ and $\mathcal{U} /(\mathcal{U} \chi) \rightarrow F\left(M^{\prime}, M^{\prime}\right)$ are bijective; identify ${ }_{\chi} F(\widetilde{M}, \widetilde{M})_{\chi ; 0}$ with $\mathcal{U} /(\mathcal{U} \chi) \oplus \mathcal{U} /(\mathcal{U} \chi)$ through these maps. Write

$$
\operatorname{Ann}_{\mathcal{Z}\left(\mathfrak{g}_{0}\right)} \widetilde{M}=\chi \prod_{1}^{s} \chi_{j}^{r_{j}}
$$


where $\operatorname{supp}_{\mathcal{Z}\left(\mathfrak{g}_{0}\right)}=\left\{\chi, \chi_{1}, \ldots, \chi_{s}\right\}$ and $r_{1}, \ldots, r_{s} \in \mathbb{N}^{+}$. Take $a \in \prod_{1}^{s} \chi_{j}^{r_{j}}$ such that $a=1$ modulo $\chi$. The image of $\mathcal{U} a$ in $F(\widetilde{M}, \widetilde{M})$ lies in $\chi_{\chi} F(\widetilde{M}, \widetilde{M})_{\chi ; 0}$. Since for any $u \in \mathcal{U}$ the element $u a$ acts on both $M$ and $M^{\prime}$ by the multiplication by $u$, the image $J$ of $\mathcal{U} a$ in ${ }_{\chi} F(\widetilde{M}, \widetilde{M})_{\chi ; 0}$ is equal to the diagonal copy of $\mathcal{U} /(\mathcal{U} \chi)$ inside $\mathcal{U} /(\mathcal{U} \chi) \oplus \mathcal{U} /(\mathcal{U} \chi):$

$$
J=\{(u, u) \mid u \in \mathcal{U} /(\mathcal{U} \chi)\} .
$$

Then $J \theta=\{(u,-u) \mid u \in \mathcal{U} /(\mathcal{U} \chi)\}$ and thus

$$
J+J \theta=\mathcal{U} /(\mathcal{U} \chi) \oplus \mathcal{U} /(\mathcal{U} \chi)={ }_{\chi} F(\widetilde{M}, \widetilde{M})_{\chi ; 0} .
$$

Hence

$$
{ }_{\chi} F(\widetilde{M}, \widetilde{M})_{\chi ; 0}={ }_{\chi}(A+A \theta)_{\chi ; 0} .
$$

By 4.6, this forces $F(\widetilde{M}, \widetilde{M})=A+A \theta$.

We have shown that $F(\widetilde{M}, \widetilde{M})=\operatorname{Im} \tilde{\mathcal{U}} \oplus \theta \operatorname{Im} \tilde{\mathcal{U}}$ provided $\widetilde{M}$ is simple. Take an arbitrary Verma module $\widetilde{M}$ satisfying $\widetilde{\chi} \widetilde{M}=0$. The module $\widetilde{M}$ contains a simple Verma submodule $\widetilde{M^{\prime}}$ since $\widetilde{\mathcal{U}}$ is a domain. Let $E$ be an ad $\mathfrak{g}$-submodule of $\widetilde{\mathcal{U}}$ such that the restriction of the natural map $f^{\prime}: \widetilde{\mathcal{U}} \rightarrow F\left(\widetilde{M^{\prime}}, \widetilde{M^{\prime}}\right)$ to $E$ provides a bijection $E \rightarrow \operatorname{Im} f^{\prime}$. Then $F\left(\widetilde{M}^{\prime}, \widetilde{M}^{\prime}\right)=f^{\prime}(E) \oplus f^{\prime}(E) \theta$. The restriction of the natural map $f: \widetilde{\mathcal{U}} \rightarrow F(\widetilde{M}, \widetilde{M})$ to $E$ provides a bijection $E \rightarrow \operatorname{Im} f$ since $\operatorname{ker} f=\operatorname{ker} f^{\prime}=\widetilde{\mathcal{U}} \widetilde{\chi}$. Suppose that $f\left(u_{1}\right)=f\left(u_{2}\right) \theta$ for some $u_{1}, u_{2} \in E$; then $\left(u_{1}-u_{2}\right) \widetilde{M}_{0}=0$ and so $f^{\prime}\left(u_{1}\right)=f^{\prime}\left(u_{2}\right) \theta$ which implies $u_{1}=u_{2}=0$ since $f^{\prime}(E) \cap f^{\prime}(E) \theta=0$. Therefore $f(E) \cap f(E) \theta=0$. Thus $F(\widetilde{M}, \widetilde{M})$ contains $f(E) \oplus f(E) \theta$. A Joseph's reasoning based on the use of GK-dimension shows that the map $F\left(\widetilde{M^{\prime}}, \widetilde{M}^{\prime}\right) \rightarrow F\left(\widetilde{M^{\prime}}, \widetilde{M}\right)$ is bijective and the map $F(\widetilde{M}, \widetilde{M}) \rightarrow F\left(\widetilde{M}^{\prime}, \widetilde{M}\right)$ is injective (both maps are induced by the embedding $\widetilde{M}^{\prime}$ to $\left.\widetilde{M}\right)$ - see [J2], 8.3.9, or [G2], 9.2. In particular, $F(\widetilde{M}, \widetilde{M})$ is isomorphic to an ad $\mathfrak{g}$-submodule of $F\left(\widetilde{M}^{\prime}, \widetilde{M}^{\prime}\right)$. On the other hand, $F(\widetilde{M}, \widetilde{M})$ contains $f(E) \oplus f(E) \theta$ which is isomorphic, as an ad $\mathfrak{g}_{0}$-module, to $F\left(\widetilde{M^{\prime}}, \widetilde{M^{\prime}}\right)$. Since the multiplicity of each finite-dimensional $\mathfrak{g}_{0}$-module in $F\left(\widetilde{M}^{\prime}, \widetilde{M^{\prime}}\right)$ is finite, one concludes that $F(\widetilde{M}, \widetilde{M})=f(E) \oplus f(E) \theta=\operatorname{Im} f \oplus \theta \operatorname{Im} f$ as required.

\section{ACKNowledgments}

The results of this paper were obtained when I was a visitor at MSRI and at Max-Planck Institut für Mathematik at Bonn. I express my gratitude to these institutions for the hospitality and excellent working conditions. I wish to thank V. Serganova and I. Penkov for helpful discussions.

\section{REFERENCES}

[BF] A. D. Bell, R. Farnsteiner, On the theory of Frobenius extensions and its application to Lie superalgebras, Trans. AMS 335 (1993) no.1, 407-424. MR 93c:17049

[BL] J. Bernstein, V. Lunts, A simple proof of Kostant's theorem that $\widetilde{\mathcal{U}}$ is free over its center, Amer. J. Math. 118 (1996), no.5, 979-987. MR 97h:17012

[BZV] M. Bershadsky, S. Zhukov, A. Vaintrob, $\mathfrak{p s l}(n \mid n)$ sigma model as a conformal field theory, Nuclear Phys. B 559 (1999), no.1-2, 205-234. MR 2001b:81128

[D] M. Duflo, Construction of primitive ideals in an enveloping algebra, in: I. M. Gelfand, ed., Publ. of 1971 Summer School in Math., Janos Bolyai Math. Soc., Budapest, 77-93. MR 53:3045 
[G1] M. Gorelik, On the ghost centre of Lie superalgebras, Ann. Inst. Fourier. 50 (2000), no.6, $1745-1764$.

[G2] M. Gorelik, Annihilation Theorem and Separation Theorem for basic classical Lie superalgebras, preprint MSRI 2000-019, math.RA/0008143.

[GL1] M. Gorelik, E. Lanzmann, The minimal primitive spectrum of the enveloping algebra of the Lie superalgebra osp $(1,2 l)$, Adv. in Math. 154 (2000), no.2, 333-366. MR 2001g:17023

[J1] A. Joseph, Kostant's problem, Goldie rank and the Gelfand-Kirillov conjecture, Invent. Math. 56 (1980), 193-204. MR 82f:17008

[J2] A. Joseph, Quantum groups and their primitive ideals, Springer, 1995. MR 96d:17015

[K1] V. G. Kac, Lie superalgebras, Adv. in Math. 26 (1977), 8-96. MR 58:5803

[K2] V. G. Kac, Characters of typical representations of Lie superalgebras, Comm. Alg. 5 (1977), 889-997. MR 56:3075

[K3] V. G. Kac, Representations of classical Lie superalgebras, Lecture Notes in Math., SpringerVerlag, Berlin, 676 (1978), 597-626. MR 80f:17006

[Ko] B. Kostant, Lie group representations on polynomial rings, Amer. J. Math. 85 (1963), 327-404. MR 28:1252

[M1] I. M. Musson, On the center of the enveloping algebra of a classical simple Lie superalgebra, J. of Algebra 193 (1997), 75-101. MR 98k:17012

[P] I. Penkov, Generic representations of classical Lie superalgebras and their localization, Monatshefte f. Math. 118 (1994), 267-313. MR 95k:17011

[PS1] I. Penkov, V. Serganova, Representation of classical Lie superalgebras of type I, Indag. Mathem. N.S. 3 (4) (1992), 419-466. MR 93k:17006

[PS2] I. Penkov, V. Serganova, Generic irreducible representations of finite-dimensional Lie superalgebras, International J. Math., Vol. 5, No. 3 (1994), 389-419. MR 95c:17015

[S1] A. N. Sergeev, Invariant polynomial functions on Lie superalgebras, C.R. Acad. Bulgare Sci. 35 (1982), no.5, 573-576. MR 84h:17015

Department of Mathematics, Weizmann Institute of Science, Rehovot 76100, Israel

E-mail address: gorelik@wisdom.weizmann.ac.il 